\title{
Evaluating the Predictability of Stock Market Returns via STARX-Tree Models
}

\author{
Camila Epprecht, Álvaro Veiga \\ Pontifical Catholic University of Rio de Janeiro, Rio de Janeiro, Brasil
}

\begin{abstract}
The goal of this paper is to evaluate the predictability of a stock market using the one-step-ahead forecasts of the returns as a signal for automatic trading. The paper presents a proposal of a multiple regime model that combines aspects from STAR models, and decision trees. The resulting model, so-called STARX-Tree, is a regression tree with smooth transition and linear ARX models fitted in the terminal nodes. The methodology was tested on 23 stocks of the U.S. stock market. The forecasting model is evaluated through statistical and financial measures and compared to the random walk model, the naive approach, the neural networks and the linear ARX model. The results pointed out that the STARX-Tree model outperforms the comparative models under the financial criterion.

Keywords: nonlinear models, regression tree, STARX-Tree, heteroscedastic, realized volatility, automatic trading
\end{abstract}

\section{Introduction}

Over the last years, many nonlinear models have been proposed to time series analysis, in classical econometrics where multiple regime models such as (MR)STAR (Granger \& Teräsvirta, 1993) have received much attention and in machine learning theory where artificial neural networks and recursive partitioning methods, specially decision trees, are often used.

This paper presents a construction of a nonlinear regression model with external variables that combines aspects of two well-known methodologies: Classification and Regression Trees (CART) discussed in Breiman, Friedman, Olshen, and Stone (1984) and the Smooth Transition Autoregression (STAR) presented in Granger and Teräsvirta (1993).

STARX-Tree models (Tree-structured Smooth Transition Autoregression with external variables) are switching regime formulations where a different ARX model is active at each instant, depending on the current "state of the system", this one defined by a vector of observable variables, called the transition variables. The decision tree that defines the switching scheme has the advantage of being interpretable as long as the tree is not too large. With this formulation, it is possible to verify which variables are determinant to induce a change in regime and at what level. Smooth transition tree models have been applied successfully in return and realized volatility forecasting (Da Rosa, Veiga, \& Medeiros, 2008; Scharth \& Medeiros, 2009).

Camila Epprecht, MSc, Department of Electric Engineering, Pontifical Catholic University of Rio de Janeiro. Álvaro Veiga, Ph.D., DEE and Instituto de Atuária e Risco IAPUC, Pontifical Catholic University of Rio de Janeiro. Correspondence concerning this article should be addressed to Camila Epprecht, DEE, PUC-Rio, Rua Marqûes de São Vicente 225, 22453-900-Gávea-Rio de Janeiro-RJ-Brasil. E-mail: camila@ele.puc-rio.br. 
In this paper, we propose an application of the STARX-Tree formulation to automatic trading based on the simultaneous modelling of daily returns and realized volatilities. Variable selection for both parts of the model is completely automatic, implemented through a series of statistical tests. The one-day-ahead forecasts of return and volatility are then used to produce buy-sell signals and estimate the best leverage level under a given risk constrain. We also use the realized volatility to transform the financial series into homoscedastic series.

The methodology was tested on 23 stocks negotiated at the NYSE and compared to the random walk model (the most difficult to beat), the naive approach, the neural networks and the linear ARX model. The forecasting models are evaluated through statistical and financial measures. The explanatory variables include past returns, past volatilities, trading volume and some technical analysis indexes. The results show that the STARX-Tree formulation outperforms traditional models and is a competitive alternative to neural networks. Also, the analysis of the set of transition variables gives some interesting clues about the effect of volatility changes and technical analysis indicators on returns.

This paper is divided as follows: Section 2 describes the STARX-Tree methodology, including its interpretation and aspects of its modeling cycle. Section 3 presents an application of the STARX-Tree model on the U.S. stock market. The predictive performance of the model is evaluated by statistical and financial measures and compared with other models. We examine the statistical significance of the model's forecasts by employing a modified version of the Diebold-Mariano test (Harvey, Leybourne, \& Newbold, 1997). Finally, section 4 concludes.

\section{The STARX-Tree Model}

The STARX-Tree model is a combination of the STAR (Smooth Transition Autoregression) and CART (Classification and Regression Tree) formulations.

The STAR model, proposed by Chan and Tong (1986), Granger and Teräsvirta (1993) and Teräsvirta (1994), can be seen as a linear autoregressive model in which the coefficients are determined by the position of the explanatory variables vector in the transition space. The main issue is how to describe the relationship between the transition space and the coefficients. Also, STAR model inherits important properties from the linear models and inferential tools for specification, estimation and diagnostic checks.

Tree-structured regression and classification methods, like CART, proposed by Breiman et al. (1984), represent a sequence of rules by a binary decision tree with the output estimates or classification on the leaves. If the tree is not too large, the interpretation of the rules is immediate.

The STARX-Tree model, which is a generalization of the STR-Tree proposed by Da Rosa et al. (2008), is a STAR model where the relationship between the transition space and the coefficients is induced by a decision tree. The root node splits the transition space into two semi-spaces. Child nodes can then split each semi-space subsequently. In the end, each semi-space will be associated with an ARX model, corresponding to the leaves of the tree.

\section{Mathematical Formulation}

In the STARX-Tree model, the sharp splits of the CART formulation are replaced by smooth transitions represented by the logistic functions: 


$$
G(w ; \gamma, c)=\frac{1}{1+e^{-\gamma(w-c)}}
$$

where slope parameter $\gamma$ controls the smoothness of the logistic function. The special case of the usual regression tree model is obtained when the slope parameter approaches infinity. The parameter $c$ is called the location parameter. The variable $w$ is called the transition variable.

The nodes of the tree are numbered as follows: the root node is at position $j=0$. A parent node at position $j$ generates left- and right-child nodes at positions $2 j+1$ and $2 j+2$, respectively. Furthermore, let $\mathbb{J}$ and $\mathbb{T}$ be the sets of indexes of the parent and terminal nodes, respectively.

Let $\mathrm{x}_{t}=\left\{\mathrm{x}_{1, t}, \ldots, \mathrm{x}_{q, t}\right\}$ be a set of $q$ candidate transition variables. Also, define the set $\left(\mathrm{z}_{t} \subseteq \mathrm{x}_{t}\right)$ with $\mathrm{z}_{t} \in$ $\mathbb{R}^{p}, p<q$, of variables to be included in the ARX models and define $\tilde{\mathrm{z}}_{t}=\left(1, \mathrm{z}_{t}\right)^{\prime}$. A parametric model defined by the function $H_{\mathbb{D} T}\left(\mathrm{x}_{t} ; \psi\right): \mathbb{R}^{q+1} \rightarrow \mathbb{R}$ indexed by the vector of parameters $\psi$ is called STARX-Tree (Smooth Transition Autoregression Tree with external variables) if:

$$
H_{\mathbb{V} \mathbb{T}}\left(\mathrm{x}_{t} ; \psi\right)=\sum_{i \in \mathbb{T}} \beta_{i}^{\prime} \tilde{z}_{t} B_{\mathbb{}}\left(\mathrm{x}_{t} ; \theta_{i}\right)
$$

where

$$
B_{\mathbb{}}\left(\mathrm{x}_{t} ; \theta_{i}\right)=\prod_{j \in \mathbb{I}} G\left(x_{s_{j}, t} ; \gamma_{j}, c_{j}\right)^{\frac{n_{i, j}\left(1+n_{i, j}\right)}{2}}\left[1-G\left(x_{s_{j}, t} ; \gamma_{j}, c_{j}\right)\right]^{\left(1-n_{i, j}\right)\left(1+n_{i, j}\right)}
$$

and

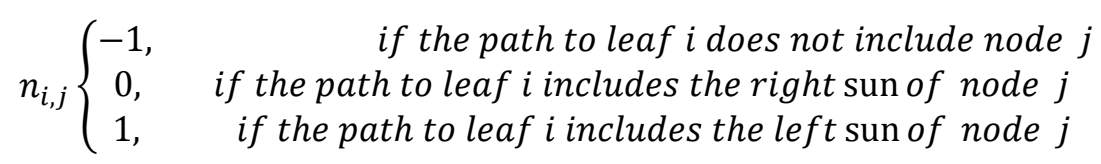

Let $\mathbb{I}_{i}$ be the subset of $\mathbb{J}$ containing the indexes of the parent nodes that form the path to leaf (terminal node) $i \in \mathbb{T}$. Then, $\theta_{i}$ is the vector containing all the parameters $\left(\gamma_{k}, c_{k}\right)$ such that $k \in \mathbb{I}_{i}$.

$G\left(\right.$.) is the logistic function defined in equation (1). The functions $B_{\rrbracket i}, 0<B_{\rrbracket i}<1$, are known as the membership functions. Note that $\sum_{j \in \mathbb{D}} B_{\mathbb{}}\left(\mathrm{x}_{t} ; \theta_{j}\right)=1, \forall \mathrm{x}_{t} \in \mathbb{R}^{q}$.

As our goal is to model financial series, which are heteroscedastic, we defined the following STARX-Tree model:

$$
\begin{gathered}
y_{t}=H_{\mathbb{T}}\left(\mathrm{x}_{t} ; \psi\right)+\varepsilon_{t}=\sum_{i \in \mathbb{T}} \beta_{i}^{\prime} \tilde{\mathrm{z}}_{t} B_{\mathbb{}}\left(\mathrm{x}_{t} ; \theta_{i}\right)+\varepsilon_{t} \\
\varepsilon_{t}=h_{t}^{1 / 2} \eta_{t}
\end{gathered}
$$

where $\eta_{t} \sim \operatorname{NID}(0,1)$ and $h_{t}^{1 / 2}$ is the volatility of the time series.

Once we have the daily series of realized volatilities of financial series, we can divide both sides of equation (5) by the realized volatility.

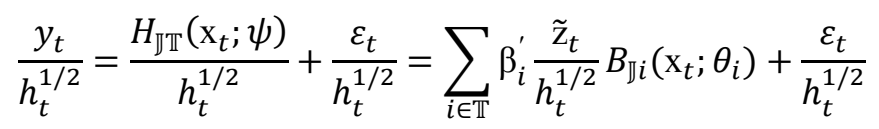

We can rewrite equation (6) as:

$$
y_{t}^{*}=H_{\mathbb{T}}^{*}\left(\mathrm{x}_{t} ; \psi\right)+\eta_{t}=\sum_{i \in \mathbb{T}} \beta_{i}^{\prime} \tilde{\mathrm{z}}_{t}^{*} B_{\mathbb{}}\left(\mathrm{x}_{t} ; \theta_{i}\right)+\eta_{t}
$$


In equation (7), $\eta_{t} \sim \operatorname{NID}(0,1)$ and we have a homoscedastic model for the series $y_{t}{ }^{*}$. After the estimation we obtain the forecast of $y_{t}{ }^{*}$ :

$$
\hat{y}_{t}^{*}=H_{\mathbb{T}}^{*}\left(\mathrm{x}_{t} ; \hat{\psi}\right)=\sum_{i \in \mathbb{T}} \hat{\beta}_{i}^{\prime} \tilde{z}_{t}^{*} B_{\rrbracket i}\left(\mathrm{x}_{t} ; \hat{\theta}_{i}\right)
$$

If we multiply both sides of the equation (8) by $h_{t}^{1 / 2}$, we have equation (9) that gives the forecast in the original scale:

$$
\hat{y}_{t}=H_{\mathbb{T}}\left(\mathrm{x}_{t} ; \hat{\psi}\right)=\sum_{i \in \mathbb{T}} \hat{\beta}_{i}^{\prime} \tilde{z}_{t} B_{\rrbracket i}\left(\mathrm{x}_{t} ; \hat{\theta}_{i}\right)
$$

Therefore, after estimating the model in equation (8), we can use the parameters estimate on the original series to obtain the original series $y_{t}$ estimate.

\section{Model Building}

The modeling cycle of the STARX-Tree model involves two steps: specification and estimation.

The specification consists of the choice of relevant variables for the linear ARX models in the leaves and the sequential application of statistical hypothesis tests to select both the transition variables and nodes to be split. The possible candidate variables may be a set of external variables and/or a set of lags of the dependent variable. The selection of the node to be split and transition variable will be carried out by a sequence of Lagrange Multiplier (LM) tests following the ideas originally presented in Luukkonen, Saikkonen, and Teräsvirta (1988).

The estimation of the parameters of the model will be carried out after the inclusion of each new transition variable by nonlinear least squares (NLS) which is equivalent to quasi-maximum likelihood estimation.

Following the "specific-to-general" principle, we start the cycle from the root node (depth 0 ) and the general steps are:

- Selection of the relevant variables for the ARX model;

- Specification of the model by selecting in the depth $d$, using LM test, a node to be split (if not in the root node) and a transition variable;

- Estimation of the parameters.

\section{Model Estimation}

The STARX-Tree model in equation (2) is composed by the parameters vector $\psi$, such that $\psi=$ $\left(\beta_{1}, \ldots, \beta_{K}, \theta_{1}^{\prime}, \ldots, \theta_{K}^{\prime}\right)^{\prime}$, where $\beta_{i}$ is the vector of the linear parameters, and $\theta_{i}^{\prime}$ is the vector of the nonlinear parameters $\left(\gamma_{j}, c_{j}\right)$ of the terminal node $i$, generate from the node $j$.

As the errors of the model $\eta_{t}$ are $\operatorname{NID}(0,1)$, presented in equation (7), we can use de maximum likelihood principle, that under the hypothesis of normality, is equivalent to nonlinear least squares (NLS). The quasi-maximum likelihood estimator (QML) is given by:

$$
\widehat{\psi}=\underset{\psi \in \Psi}{\operatorname{argmin}} \frac{1}{T} \sum_{t=1}^{T} q_{t}(\psi)=\underset{\psi \in \Psi}{\operatorname{argmin}}\left\{\frac{1}{T} \sum_{t=1}^{T}\left[y_{t}-H_{\mathbb{T}}\left(\mathrm{x}_{t} ; \psi\right)\right]^{2}\right\}
$$

Conditionally on the knowledge of the nonlinear parameters $\theta_{i}$ in equation (2), $i=1, \ldots K$, the model in equation (2) becomes a linear regression and we can estimate the vector $\beta=\left(\beta_{1}, \ldots, \beta_{K}\right)^{\prime}$ by ordinary least squares (OLS). This procedure is called concentrated least squares. 
The parameters $\theta_{i}$ are estimated conditionally on $\beta$ by applying the nonlinear least squares, which uses the Levenberg-Marquadt algorithm (nonlinear optimization procedure) and completes the $i$ th iteration. As the NLS algorithm is sensitive to the choice of starting values, we suggest the use of a grid of possible starting values.

\section{Specification Algorithm}

In order to achieve the final tree model, we perform a sequence of $n$ correlated LM type tests of hypothesis in which $n$ is a random variable. We test the null hypothesis of linearity against the alternative of a STARX-type nonlinearity.

Under the null, and assuming that $E\left(\left|x_{l t}\right| \delta\right)<\infty, l=1, \ldots, q$, for any $\delta>6$, and $\eta_{t} \sim \operatorname{NID}\left(0, \sigma^{2}\right)$, the LM type statistic:

$$
M L=\frac{1}{\hat{\sigma}^{2}} \sum_{t=1}^{T} \hat{u}_{t} \hat{v}_{t}^{\prime}\left\{\sum_{t=1}^{T} \hat{v}_{t} \hat{v}_{t}^{\prime}-\sum_{t=1}^{T} \hat{v}_{t} \hat{\mathrm{h}}_{t}^{\prime}\left(\sum_{t=1}^{T} \hat{\mathrm{h}}_{t} \hat{\mathrm{h}}_{t}^{\prime}\right)^{-1} \sum_{t=1}^{T} \hat{\mathrm{h}}_{t} \hat{v}_{t}\right\}^{-1} \sum_{t=1}^{T} \hat{v}_{t} \hat{u}_{t}
$$

where $\hat{u}_{t}=y_{t}-H_{\mathbb{J T}}\left(x_{t} ; \hat{\psi}\right)$ is the estimated residuals under the null:

$$
\begin{gathered}
\hat{\mathrm{h}}_{t}=\left.\frac{\partial H_{\mathbb{T} T}\left(\mathrm{x}_{t} ; \psi\right)^{\prime}}{\partial \psi}\right|_{\psi=\widehat{\psi}} \\
=\left[\tilde{\mathrm{z}}_{t} B_{\mathbb{\nabla} i_{1}}\left(\mathrm{x}_{t} ; \psi\right), \ldots, \tilde{\mathrm{z}}_{t} B_{\mathbb{\nabla} i_{K}}\left(\mathrm{x}_{t} ; \psi\right),\left.\quad \beta_{i_{1}}^{\prime} \tilde{\mathrm{z}}_{t} \frac{\partial B_{\mathbb{D} i_{1}}\left(\mathrm{x}_{t} ; \theta_{i_{1}}\right)}{\partial \theta_{i_{1}}}\right|_{\theta_{i_{1}}=\widehat{\theta}_{i_{1}}}, \ldots,\left.\beta_{i_{K}}^{\prime} \tilde{\mathrm{z}}_{t} \frac{\partial B_{\mathbb{N} i_{K}}\left(\mathrm{x}_{t} ; \theta_{i_{K}}\right)}{\partial \theta_{i_{K}}}\right|_{\theta_{i_{K}}=\widehat{\theta}_{i_{K}}}\right]^{\prime}
\end{gathered}
$$

and $\hat{\mathrm{v}}_{\mathrm{t}}=\left[\tilde{\mathrm{z}}_{t} B_{\mathbb{\nabla} i^{*}}\left(\mathrm{x}_{\mathrm{t}} ; \hat{\theta}_{\mathrm{i}^{*}}\right) x_{i^{*} t}, \tilde{\mathrm{z}}_{t} B_{\mathbb{\nabla} i^{*}}\left(\mathrm{x}_{\mathrm{t}} ; \hat{\theta}_{\mathrm{i}^{*}}\right) x_{i^{*} t}^{2}, \tilde{\mathrm{z}}_{t} B_{\mathbb{I} i^{*}}\left(\mathrm{x}_{\mathrm{t}} ; \hat{\theta}_{\mathrm{i}^{*}}\right) x_{i^{*} t}^{3}\right]^{\prime}$, has an asymptotic $\chi^{2}$ distribution with $m=3(p+1)$ degrees of freedom.

From the assumption of normality of the error term, the information matrix is a diagonal block, and thus we can assume that the error variance is fixed. The test can be carried out according to the following steps:

Estimate model (2) with $K$ regimes. To do so, we regress the residuals $\hat{u}_{t}$ on $\hat{\mathrm{h}}_{t}$ and compute the sum of squared residuals $S S R_{0}=\sum_{t=1}^{T} \tilde{u}_{t}^{2}$. The new residuals $\tilde{u}_{t}$ are orthogonal to $\hat{h}_{t}$.

Regress de $\tilde{u}_{t}$ on $\hat{\mathrm{h}}_{t}$ and $\hat{v}_{\mathrm{t}}$. Compute the sum of squared residuals $S S R_{1}=\sum_{t=1}^{T} \hat{v}_{t}^{2}$.

Compute the $\chi^{2}$ statistic:

$$
L M_{\chi^{2}}=T \frac{S S R_{0}-S S R_{1}}{S S R_{0}}
$$

or the $F$ version of the test:

$$
L M_{F}=\frac{\left(S S R_{0}-S S R_{1}\right) / m}{S S R_{1} /(T-l-m)}
$$

where $l=(q+2) h+p+1$.

Under $\mathrm{H}_{0}, L M_{\chi^{2}}$ has an asymptotic $\chi^{2}$ distribution with $m$ degrees of freedom, and $L M_{F}$ is approximately $F$-distributed with $m$ and $T-l-m$ degrees of freedom. $T$ is the number of observations.

According to the principle of tree-complexity as function of the number of terminal nodes, the biggest 
concern is not to decide erroneously for splitting a node during the sequence of the LM tests. Due to multiplicity from repeated significance testing, we have to control the overall type I error under the risk of an overstatement of the significance of the results (i.e., more splits are reported to be significant than it should be). To remedy this situation, we adopt the procedure of decrease the significance level of the test as the tree grows.

Let $\alpha$ be the significance level of the first test. For the $n$th test in the sequence, if it is performed in the $d$ th depth, the significance level is:

$$
\alpha(d, n)=\frac{\alpha}{n^{d}}
$$

By forcing the test to be more rigorous in deeper depths, we create a procedure that diminishes the importance of using post-pruning techniques. For each split, the estimation of the parameters is done, and the final model is specified.

\section{Application to the U.S. Stock Market}

In this section, we evaluate the STARX-Tree methodology with an application to financial data. The aim is to use the one-step-ahead forecasts of the returns as a signal for automatic trading in the U.S. Stock Market. If it is positive, a buying position is taken, otherwise the trader stays out of the market.

We compare the statistical and financial results of the STARX-Tree methodology to several methods: the naive approach, which uses the present return as a forecast for the next day's return, a random walk model, which uses the historical mean, a neural network and a linear ARX model.

\section{The Data}

For this application, we use the daily returns and daily realized volatility of 23 assets from the set of the Dow Jones Industrial Average (DJIA) index as listed in Table 1. The third column of the table gives the average number of transactions per day. The data are obtained from the NYSE TAQ (Trade and Quote) database and used by Hillebrand and Medeiros (2010). The sample period covers 3-Jan.-1995 to 30-Dec.-2005. We use 80\% of the data for the specification and estimation of the models in-sample (training), and the final $20 \%$ for the forecast out-of-sample, according to Table 2 .

In calculating daily realized volatility, we employ the realized kernel estimator with modified Tukey-Hanning weights of Barndorff-Nielsen, Hansen, Lunde, and Shephard (2008), which is a consistent estimators and robust to microstructure noise.

The series of returns are then transformed by dividing each return by its realized volatility $\left(h_{t}^{1 / 2}\right)$, which gives homoscedastic series as shown in equation (15).

$$
y_{t}^{*}=\frac{y_{t}}{h_{t}^{1 / 2}}
$$

The returns were obtained from the closing price $\left(p_{t}\right)$ of the asset in two consecutive days according to equation (16).

$$
y_{t}=\ln \left(\frac{p_{t}}{p_{t-1}}\right)
$$


Table 1

Data Description

\begin{tabular}{llc}
\hline Symbol & Stock & Transactions per day \\
\hline AA & Alcoa Inc. & 934 \\
AIG & American International Group Inc. & 1,529 \\
AXP & American Express Co. & 1,252 \\
BA & Boeing Co. & 1,390 \\
CAT & Caterpillar Inc. & 1,024 \\
DD & Du Pont de Nemours \& Co. & 1,261 \\
DIS & Walt Disney Co. & 1,726 \\
GE & General Eletrics Co. & 3,879 \\
GM & General Motors Corp. & 1,347 \\
HD & Home Depot Inc. & 2,322 \\
HOM & Honeywell International Inc. & 895 \\
IBM & International Business Machines Corp. & 2,820 \\
JNJ & Johnson \& Johnson & 1,838 \\
JPM & JPMorgan Chase \& Co. & 1,663 \\
KO & Coca-Cola Co. & 1,531 \\
MCD & McDonald's Corp. & 1,209 \\
MMM & 3M Corp. & 1,118 \\
MO & Altria Group Inc. & 1,947 \\
MRK & Merck \& Co. Inc. & 2,073 \\
PFE & Pfizer Inc. & 3,315 \\
PG & Procter and Gamble Co. & 1,510 \\
UTX & United Technologies Corp. & 949 \\
WMT & Wal-Mart Stores Inc. & 2,281 \\
\hline
\end{tabular}

Table 2

Training and Test Periods

\begin{tabular}{llrr}
\hline Period description & Number of observations & Beginning & End \\
\hline Total & 2,721 & 15-Mar.-95 & 30 -Dec.-05 \\
Training (in-sample) & 2,177 & 15-Mar.-95 & 3-Nov.-03 \\
Test set (out-of-sample) & 544 & 4-Nov.-03 & 30-Dec.-05 \\
\hline
\end{tabular}

Realized volatility ${ }^{2}$ is defined as the sum of the $n_{t}$ squared intra-day returns $r_{t, i}$ of day $t$ :

$$
h_{t}^{1 / 2}=\sqrt{\sum_{i=1}^{n_{t}} r_{t, i}^{2}}
$$

Table 3 shows some descriptive statistics of the returns $\left(y_{t}\right.$ and $\left.y_{t}{ }^{*}\right)$. From this table we can confirm some stylized facts such as the excess of kurtosis and asymmetry. The Jarque-Bera test confirms, at 0.01 significance

\footnotetext{
${ }^{2}$ In practice, high frequency measures are contaminated by microstructure noise such as bid-ask bounce, asynchronous trading, infrequent trading, price discreteness, among others (see Biais, Glosten, \& Spatt, 2005). Ignoring the remaining measurement error, this ex post volatility measure can modeled as an "observable" variable, in contrast to the latent variable models (see Andersen, Bollerslev, Diebold, \& Labys, 2003; Barndorff-Nielsen \& Shephard, 2002) for the theoretical foundations of realized volatility. Several recent papers have proposed corrections to estimation of RV in order to take the microstructure noise into account (see McAleer \& Medeiros, 2008 for a review).
} 
level, that the returns are not normally distributed. Note that the residuals of $y_{t}{ }^{*}$ are $\operatorname{NID}(0,1)$, as in equation (7). However, the variance of the series $y_{t}{ }^{*}$ is higher than 1 . This fact can be explained from possible errors on the realized volatility data.

Table 3

Descriptive Measures of Returns

\begin{tabular}{|c|c|c|c|c|c|c|c|}
\hline Asset & Series & Mean & Variance & Asymmetry & Curtosis & Jarque-Bera & $p$-value \\
\hline \multirow[t]{2}{*}{ AA } & $y_{t}$ & 0.04 & 4.85 & 0.24 & 5.81 & 919.62 & 0.00 \\
\hline & $y_{t}{ }^{*}$ & 0.03 & 1.71 & 0.49 & 6.55 & $1,538.08$ & 0.00 \\
\hline \multirow[t]{2}{*}{ AIG } & $y_{t}$ & 0.00 & 5.41 & -5.82 & 101.74 & $1.12 \mathrm{E}+06$ & 0.00 \\
\hline & $y_{t}{ }^{*}$ & 0.02 & 2.72 & -11.24 & 286.02 & $9.12 \mathrm{E}+06$ & 0.00 \\
\hline \multirow[t]{2}{*}{ AXP } & $y_{t}$ & 0.06 & 4.59 & -0.12 & 6.67 & $1,529.82$ & 0.00 \\
\hline & $y_{t}{ }^{*}$ & 0.05 & 1.58 & -0.28 & 6.67 & $1,561.41$ & 0.00 \\
\hline \multirow[t]{2}{*}{$\mathrm{BA}$} & $y_{t}$ & 0.04 & 4.43 & -0.66 & 11.15 & $7,721.22$ & 0.00 \\
\hline & $y_{t}{ }^{*}$ & 0.05 & 1.48 & 0.00 & 3.13 & 1.76 & 0.42 \\
\hline \multirow[t]{2}{*}{ CAT } & $y_{t}$ & 0.06 & 4.27 & -0.08 & 5.60 & 768.92 & 0.00 \\
\hline & $y_{t}{ }^{*}$ & 0.05 & 1.60 & 0.05 & 3.09 & 2.19 & 0.33 \\
\hline \multirow[t]{2}{*}{$\mathrm{DD}$} & $y_{t}$ & 0.02 & 3.45 & -0.02 & 6.15 & $1,124.86$ & 0.00 \\
\hline & $y_{t}{ }^{*}$ & 0.03 & 1.32 & 0.13 & 3.11 & 8.71 & 0.01 \\
\hline \multirow[t]{2}{*}{ DIS } & $y_{t}$ & 0.01 & 4.53 & -0.21 & 11.20 & $7,628.75$ & 0.00 \\
\hline & $y_{t}{ }^{*}$ & 0.02 & 1.48 & 0.12 & 4.23 & 176.10 & 0.00 \\
\hline \multirow[t]{2}{*}{ GE } & $y_{t}$ & 0.05 & 3.26 & 0.09 & 6.42 & $1,329.53$ & 0.00 \\
\hline & $y_{t}{ }^{*}$ & 0.07 & 1.34 & 0.26 & 3.20 & 35.76 & 0.00 \\
\hline \multirow[t]{2}{*}{ GM } & $y_{t}$ & -0.03 & 4.54 & -0.27 & 9.26 & $4,462.93$ & 0.00 \\
\hline & $y_{t}{ }^{*}$ & -0.01 & 1.74 & -0.02 & 3.56 & 35.57 & 0.00 \\
\hline \multirow[t]{2}{*}{ HD } & $y_{t}$ & 0.02 & 6.37 & -3.64 & 58.01 & $3.49 \mathrm{E}+05$ & 0.00 \\
\hline & $y_{t}{ }^{*}$ & 0.04 & 2.34 & -8.35 & 205.13 & $4.66 \mathrm{E}+06$ & 0.00 \\
\hline \multirow[t]{2}{*}{$\mathrm{HOM}$} & $y_{t}$ & 0.02 & 5.29 & -0.43 & 12.79 & $10,934.28$ & 0.00 \\
\hline & $y_{t}{ }^{*}$ & 0.05 & 1.78 & 0.37 & 6.73 & 1632.07 & 0.00 \\
\hline \multirow[t]{2}{*}{ IBM } & $y_{t}$ & 0.05 & 4.61 & -0.20 & 11.79 & $8,761.32$ & 0.00 \\
\hline & $y_{t}{ }^{*}$ & 0.06 & 1.60 & 0.17 & 3.96 & 117.79 & 0.00 \\
\hline \multirow[t]{2}{*}{ JNJ } & $y_{t}$ & 0.05 & 2.41 & -0.49 & 11.42 & $8,134.61$ & 0.00 \\
\hline & $y_{t}{ }^{*}$ & 0.05 & 1.31 & 0.11 & 3.10 & 6.68 & 0.04 \\
\hline \multirow[t]{2}{*}{ JPM } & $y_{t}$ & 0.03 & 4.76 & 0.13 & 9.32 & $4,530.05$ & 0.00 \\
\hline & $y_{t}{ }^{*}$ & 0.04 & 1.54 & 0.12 & 3.02 & 6.22 & 0.04 \\
\hline
\end{tabular}


(Table 3 continued)

\begin{tabular}{|c|c|c|c|c|c|c|c|}
\hline Asset & Series & Mean & Variance & Asymmetry & Curtosis & Jarque-Bera & $p$-value \\
\hline \multirow[t]{2}{*}{$\mathrm{KO}$} & $y_{t}$ & 0.01 & 2.74 & -0.06 & 6.97 & $1,784.91$ & 0.00 \\
\hline & $y_{t}{ }^{*}$ & 0.03 & 1.29 & 0.13 & 3.46 & 30.53 & 0.00 \\
\hline \multirow[t]{2}{*}{ MCD } & $y_{t}$ & 0.03 & 3.23 & -0.08 & 7.36 & 2149.39 & 0.00 \\
\hline & $y_{t}{ }^{*}$ & 0.04 & 1.22 & 0.15 & 3.51 & 40.06 & 0.00 \\
\hline \multirow[t]{2}{*}{ MMM } & $y_{t}$ & 0.04 & 2.53 & 0.17 & 6.19 & 1165.08 & 0.00 \\
\hline & $y_{t}{ }^{*}$ & 0.02 & 1.37 & -0.07 & 4.61 & 296.14 & 0.00 \\
\hline \multirow[t]{2}{*}{ MO } & $y_{t}{ }_{*}$ & 0.05 & 4.14 & -0.26 & 10.05 & 5661.79 & 0.00 \\
\hline & $y_{t}{ }^{*}$ & 0.09 & 1.46 & 0.01 & 3.65 & 47.99 & 0.00 \\
\hline \multirow[t]{2}{*}{ MRK } & $y_{t}$ & 0.02 & 3.64 & -1.69 & 31.48 & $9.31 \mathrm{E}+04$ & 0.00 \\
\hline & $y_{t}{ }^{*}$ & 0.05 & 1.46 & -0.15 & 4.50 & 262.39 & 0.00 \\
\hline \multirow[t]{2}{*}{ PFE } & $y_{t}$ & 0.04 & 3.78 & -0.16 & 5.23 & 572.05 & 0.00 \\
\hline & $y_{t}{ }^{*}$ & 0.06 & 1.49 & 0.17 & 2.91 & 13.24 & 0.00 \\
\hline \multirow[t]{2}{*}{ PG } & $y_{t}$ & 0.04 & 3.06 & -3.84 & 83.84 & $7.46 \mathrm{E}+05$ & 0.00 \\
\hline & $y_{t}{ }^{*}$ & 0.08 & 1.23 & 0.06 & 3.54 & 33.84 & 0.00 \\
\hline \multirow[t]{2}{*}{ UTX } & $y_{t}$ & 0.07 & 3.64 & -1.84 & 34.39 & $1.13 \mathrm{E}+05$ & 0.00 \\
\hline & $y_{t}{ }^{*}$ & 0.07 & 1.43 & 0.20 & 5.91 & 975.51 & 0.00 \\
\hline \multirow[t]{2}{*}{ WMT } & $y_{t}$ & 0.05 & 3.86 & 0.12 & 5.60 & 767.66 & 0.00 \\
\hline & $y_{t}{ }^{*}$ & 0.03 & 1.68 & -3.04 & 64.07 & $4.26 \mathrm{E}+05$ & 0.00 \\
\hline
\end{tabular}

\section{Explanatory Variables}

The set of explanatory variables is composed by the first 10 lags of returns $\left(y_{t-1}, \ldots, y_{t-10}\right)$, realized volatilities $\left(h_{t-1}^{1 / 2}, \ldots, h_{t-10}^{1 / 2}\right)$ and transaction volume variations between two consecutive days $\left(q_{t-1}, \ldots, q_{t-10}\right)$, first lag of accumulated returns of 2,5 and 39 days $(r 2, r 5, r 39)$, exponential moving averages prices of 12,26 and 50 days (MME12, MME26 and MME50), technical analysis indicators (MACD and ind_MM), and the variable time. The volume variation, accumulated returns, exponential moving averages and technical indicators are given by equations (18)-(22), respectively.

$$
q_{t}=\frac{t_{t}}{t_{t-1}}
$$

In equation (18), $t_{t}$ is the number of transactions at day $t$.

$$
r_{t, N}=y_{t}+\ldots+y_{t-N+1}
$$

Let $y_{t}$ be the return of day $t$. Equation (19) shows the accumulated return of $N$ days at day $t$.

$$
\begin{gathered}
M M E_{t, N}=p_{t} K+M M E_{t-1, N} *(1-K) \\
K=\frac{2}{N+1}
\end{gathered}
$$

Let $p_{t}$ be the closing price at day $t$. The exponential moving average of $N$ days at day $t$ is given by equation (20).

$$
\begin{gathered}
\text { ind_MM }{ }_{\mathrm{t}}=\frac{\mathrm{p}_{\mathrm{t}}-\mathrm{MME}_{\mathrm{t}, 50}}{\mathrm{p}_{\mathrm{t}}} \\
M A C D_{t}=M M E_{t, 12}-M M E_{t, 26}
\end{gathered}
$$


The technical analysis indicators used as explanatory variables are the Moving Average, defined in equation (21), and the Moving Average Convergence/Divergence, given by equation (22).

\section{STARX-Tree Fitted Models}

The STARX-Tree model was fitted to each one of the time series of transformed returns $y_{t}{ }^{*}$. The set of possible transition variables $\left(\mathrm{x}_{t}\right)$ is composed by the 39 explanatory variables. The set of regressors $\left(\widetilde{\mathrm{z}}_{t}^{*}\right)$ is composed only by the statistically significant variables on the ARX model, presented in Table 4 for each stock.

Table 4

Explanatory Variables of ARX Models and Regressors of STARX-Tree Models

\begin{tabular}{|c|c|c|c|c|c|c|c|c|c|c|c|c|c|c|c|c|c|c|c|c|c|c|c|c|}
\hline Variable & AA & AIG & AXP & BA & CAT & DD & DIS & GE & GM & HD & $\mathrm{HOM}$ & IBM & $\mathrm{JNJ}$ & JPM & $\mathrm{KO}$ & MCD & MMM & MO & MRK & PFE & PG & UTX & WMT & Total \\
\hline $\mathrm{y}_{\mathrm{t}-1}$ & & & & & & & & & & & & & & & & & & & & & & & & 0 \\
\hline $\mathrm{y}_{\mathrm{t}-2}$ & & & & & & & & & & & & & & & & & & & & & & & & 0 \\
\hline $\mathrm{y}_{\mathrm{t}-3}$ & & & & & & & & & & & & & & & & & & & & & & & & 0 \\
\hline $\mathrm{y}_{\mathrm{t}-4}$ & & & & & & & & & & & & & & & & & & & & & & & & 0 \\
\hline $\mathrm{y}_{\mathrm{t}-5}$ & & & & & & & & & & & & & & & & & & & & & & & & 0 \\
\hline $\mathrm{y}_{\mathrm{t}-6}$ & & & & & & & & & & & & & & & & & & & & $\mathrm{X}$ & & & & 1 \\
\hline $\mathrm{y}_{\mathrm{t}-7}$ & $\mathrm{X}$ & & $\mathrm{X}$ & & & & & & & & $\mathrm{X}$ & & & & & & $\mathrm{X}$ & & & & & & $\mathrm{X}$ & 5 \\
\hline $\mathrm{y}_{\mathrm{t}-8}$ & & & & & & & & & & & $\mathrm{X}$ & & & & & & & & & $\mathrm{X}$ & & & $\mathrm{X}$ & 3 \\
\hline$y_{t-9}$ & & & & $\mathrm{X}$ & & & & & & & & & & & & & & X & & & & & $\mathrm{X}$ & 3 \\
\hline $\mathrm{y}_{\mathrm{t}-10}$ & $\mathrm{X}$ & & & $\mathrm{X}$ & & & & $\mathrm{X}$ & & & $\mathrm{X}$ & & $\mathrm{X}$ & & $\mathrm{X}$ & & $\mathrm{X}$ & & & $\mathrm{X}$ & & $\mathrm{X}$ & & 9 \\
\hline $\mathrm{h}_{\mathrm{t}-1}$ & $\mathrm{X}$ & & & & & & $\mathrm{X}$ & & $\mathrm{X}$ & & & & & & & & $\mathrm{X}$ & & & & & & $\mathrm{X}$ & 5 \\
\hline$h_{t-2}$ & & & & & & & & $\mathrm{X}$ & & & & & & & & & $\mathrm{X}$ & & & $\mathrm{X}$ & & & & 3 \\
\hline$h_{t-3}$ & $\mathrm{X}$ & & $\mathrm{X}$ & & & & & & & & & & & & & & & $\mathrm{X}$ & & & & $\mathrm{X}$ & & 4 \\
\hline$h_{t-4}$ & & & & $\mathrm{X}$ & & & & & $\mathrm{X}$ & & $\mathrm{X}$ & & & & & & & & & & & & & 3 \\
\hline$h_{t-5}$ & & & & & & & & $\mathrm{X}$ & & & & & & X & & & & & & & & & $\mathrm{X}$ & 3 \\
\hline $\mathrm{h}_{\mathrm{t}-6}$ & & & & & & & & $\mathrm{X}$ & & & $\mathrm{X}$ & & & & & $\mathrm{X}$ & & & & $\mathrm{X}$ & & & & 4 \\
\hline$h_{t-7}$ & & & & & $\mathrm{X}$ & & & $\mathrm{X}$ & & & $\mathrm{X}$ & & & $X$ & & $\mathrm{X}$ & & & $\mathrm{X}$ & & & & $\mathrm{X}$ & 7 \\
\hline $\mathrm{h}_{\mathrm{t}-8}$ & & & & & $\mathrm{X}$ & & & & & & & & & & & & & X & & & & & & 2 \\
\hline$h_{t-9}$ & & & & & $\mathrm{X}$ & $\mathrm{X}$ & & & $\mathrm{X}$ & & & & & & & & & & & & & $\mathrm{X}$ & & 4 \\
\hline $\mathrm{h}_{\mathrm{t}-10}$ & & $\mathrm{X}$ & & & & & & & & & X & $\mathrm{X}$ & & & & $\mathrm{X}$ & & & & & & & & 4 \\
\hline $\mathrm{q}_{\mathrm{t}-1}$ & $\mathrm{X}$ & & & & & & & & & & & & & & & $\mathrm{X}$ & & & & & $X$ & & & 3 \\
\hline $\mathrm{q}_{\mathrm{t}-2}$ & $\mathrm{X}$ & & & $\mathrm{X}$ & $\mathrm{X}$ & & & $\mathrm{X}$ & & & & & & & & & & & & & & & & 4 \\
\hline $\mathrm{q}_{\mathrm{t}-3}$ & & & $\mathrm{X}$ & & $\mathrm{X}$ & & & & & & & & & & & & & & & & & & & 2 \\
\hline $\mathrm{q}_{\mathrm{t}-4}$ & & & $\mathrm{X}$ & & $\mathrm{X}$ & & & $\mathrm{X}$ & $X$ & & $\mathrm{X}$ & & & & & & $\mathrm{X}$ & & & & $\mathrm{X}$ & & & 7 \\
\hline $\mathrm{q}_{\mathrm{t}-5}$ & $\mathrm{X}$ & & $\mathrm{X}$ & $\mathrm{X}$ & & & & & & & $\mathrm{X}$ & & & & & & & & & & & & $\mathrm{X}$ & 5 \\
\hline $\mathrm{q}_{\mathrm{t}-6}$ & $\mathrm{X}$ & & & & & & & & & $\mathrm{X}$ & & & & & & $\mathrm{X}$ & $\mathrm{X}$ & & & $\mathrm{X}$ & & & & 5 \\
\hline $\mathrm{q}_{\mathrm{t}-7}$ & & & $\mathrm{X}$ & & & & & $\mathrm{X}$ & & & $\mathrm{X}$ & & & & & & & & & $\mathrm{X}$ & & & & 4 \\
\hline $\mathrm{q}_{\mathrm{t}-8}$ & & & $X$ & & & $\mathrm{X}$ & & & & & $\mathrm{X}$ & & & & & & & $\mathrm{X}$ & & & $X$ & & & 5 \\
\hline $\mathrm{q}_{\mathrm{t}-9}$ & & & & & $\mathrm{X}$ & & & & & & & & & & & & & & $\mathrm{X}$ & & & $\mathrm{X}$ & & 3 \\
\hline $\mathrm{q}_{\mathrm{t}-10}$ & & $\mathrm{X}$ & & & & & & & & & $\mathrm{X}$ & $\mathrm{X}$ & & & $\mathrm{X}$ & & & & & & & & $X$ & 5 \\
\hline MME12 & & & & & & & & & & & & & & & & & & & & & & & & 0 \\
\hline MME26 & & & & & & & & & & & & & & & & & & & & & & & & 0 \\
\hline MACD & & & & & & & & & & & & & & & & & & & & & & & & 0 \\
\hline MME50 & & & & & & & & & & & & & & & & & & & & $\mathrm{X}$ & & $\mathrm{X}$ & $\mathrm{X}$ & 3 \\
\hline ind_MM & & & & & & & & & & & & $\mathrm{X}$ & $\mathrm{X}$ & & & & & & & $\mathrm{X}$ & & $\mathrm{X}$ & & 4 \\
\hline $\mathrm{r} 2$ & & & & & & & & & & & & & & & & & & & & & & & & 0 \\
\hline r5 & & & & & & & & & & & & & & & & & & & & & & & & 0 \\
\hline r39 & & & & & & & & & & & $X$ & & & & & & & & & & & & $X$ & 2 \\
\hline Total & 8 & 2 & 7 & 5 & 7 & 2 & 1 & 8 & 4 & 1 & 13 & 3 & 2 & 2 & 2 & 5 & 6 & 4 & 2 & 9 & 3 & 6 & 10 & \\
\hline
\end{tabular}

Table 5 presents the selected transition variables and the number of regimes for each stock. In order to keep computational cost at a manageable level, the maximum number of regimes in each tree was set to 11 . Also, the 
analysis of the set of transition variables shows the effect of volatility changes and technical analysis indicators on returns. The selection of the time variable as a transition variable shows the presence of structural breaks in some series.

\section{Table 5}

Transition Variables and Number of Regimes in STARX-Tree Model

\begin{tabular}{|c|c|c|c|c|c|c|c|c|c|c|c|c|c|c|c|c|c|c|c|c|c|c|c|c|}
\hline Variable & AA & AIG & AXP & BA & CAT & DD & DIS & GE & GM & HD & $\mathrm{HOM}$ & IBM & $\mathrm{JNJ}$ & JPM & $\mathrm{KO}$ & MCD & MMM & $\mathrm{MO}$ & MRK & PFE & PG & UTX & WMT & Total \\
\hline $\mathrm{y}_{\mathrm{t}-1}$ & & & & & & & & & $\mathrm{X}$ & & & & & $\mathrm{X}$ & & & & & & $\mathrm{X}$ & & & $\mathrm{X}$ & 4 \\
\hline$y_{t-2}$ & & $\mathrm{X}$ & & & $\mathrm{X}$ & & & $\mathrm{X}$ & & & $\mathrm{X}$ & $\mathrm{X}$ & & $\mathrm{X}$ & $X$ & $\mathrm{X}$ & & & & & & $\mathrm{X}$ & & 9 \\
\hline $\mathrm{y}_{\mathrm{t}-3}$ & & & & & & & & & & & & & & & & & & & & & X & & & 1 \\
\hline $\mathrm{y}_{\mathrm{t}-4}$ & $\mathrm{X}$ & & & & & & & & & & & & & & $X$ & & & & & & & & & 2 \\
\hline$y_{t-5}$ & & & & & & & & & & & $\mathrm{X}$ & & & & & $\mathrm{X}$ & & & & & & & & 2 \\
\hline $\mathrm{y}_{\mathrm{t}-6}$ & & & & & $\mathrm{X}$ & & $\mathrm{X}$ & & & & & $\mathrm{X}$ & $\mathrm{X}$ & & $\mathrm{X}$ & & & & $\mathrm{X}$ & & $\mathrm{X}$ & & & 7 \\
\hline $\mathrm{y}_{\mathrm{t}-7}$ & & & & & $\mathrm{X}$ & & & $\mathrm{X}$ & & & & & & & & & & & & & & & & 2 \\
\hline $\mathrm{y}_{\mathrm{t}-8}$ & & & $\mathrm{X}$ & & & & & & $\mathrm{X}$ & & & & & & & & & & & & & & & 2 \\
\hline $\mathrm{y}_{\mathrm{t}-9}$ & & & & & & & & & & & & & & & & X & $X$ & & & & & & & 2 \\
\hline $\mathrm{y}_{\mathrm{t}-10}$ & & & & & & & $X$ & & $\mathrm{X}$ & & & & & & & & & & & & & & & 2 \\
\hline$h_{t-1}$ & & & & & & & & $\mathrm{X}$ & & & & $\mathrm{X}$ & & & & & & & & & & & & 2 \\
\hline$h_{t-2}$ & & $X$ & & & & & & & & $X$ & & & & & & & & & $\mathrm{X}$ & & X & & & 4 \\
\hline$h_{t-3}$ & & $\mathrm{X}$ & & & & & & & & & & & & & & & $\mathrm{X}$ & & & & & & & 2 \\
\hline$h_{t-4}$ & & $\mathrm{X}$ & & & & & & $\mathrm{X}$ & & & & $\mathrm{X}$ & & & & & & & & & & & $\mathrm{X}$ & 4 \\
\hline$h_{t-5}$ & & & $\mathrm{X}$ & & $\mathrm{X}$ & & & & $\mathrm{X}$ & & & & & & & & $\mathrm{X}$ & & & & & & & 4 \\
\hline $\mathrm{h}_{\mathrm{t}-6}$ & & & & & & & & & & & & & & & & & & & & $\mathrm{X}$ & & & & 1 \\
\hline $\mathrm{h}_{\mathrm{t}-7}$ & & & & & & & & & & & & & & & $\mathrm{X}$ & & & & & & & $\mathrm{X}$ & & 2 \\
\hline$h_{t-8}$ & & & $\mathrm{X}$ & & & & & & & & & & & & & & & & & & & & & 1 \\
\hline $\mathrm{h}_{\mathrm{t}-9}$ & & & & & & & & $\mathrm{X}$ & & & & & & & & & & & & & & $\mathrm{X}$ & & 2 \\
\hline$h_{t-10}$ & & X & $X$ & & & & & & & $\mathrm{X}$ & & & & & & & & & & & & & $\mathrm{X}$ & 4 \\
\hline $\mathrm{q}_{\mathrm{t}-1}$ & & & & & & & $\mathrm{X}$ & & & & $\mathrm{X}$ & & & & & & & & $\mathrm{X}$ & & & $\mathrm{X}$ & $\mathrm{X}$ & 5 \\
\hline $\mathrm{q}_{\mathrm{t}-2}$ & & & $\mathrm{X}$ & & & & & & & & $\mathrm{X}$ & & & & & & & & & & & & & 2 \\
\hline $\mathrm{q}_{\mathrm{t}-3}$ & & & & $\mathrm{X}$ & & & & & & & & & & & & & & & $\mathrm{X}$ & & & $\mathrm{X}$ & & 3 \\
\hline $\mathrm{q}_{\mathrm{t}-4}$ & & $\mathrm{X}$ & & & & $\mathrm{X}$ & & & & & & & & & & & & & & & & & & 2 \\
\hline $\mathrm{q}_{\mathrm{t}-5}$ & & & & & $\mathrm{X}$ & & & & & & & & & $\mathrm{X}$ & & & & & & & & & & 2 \\
\hline $\mathrm{q}_{\mathrm{t}-6}$ & & & & $\mathrm{X}$ & $\mathrm{X}$ & & & & & & $\mathrm{X}$ & & & & & & $\mathrm{X}$ & & & & & $\mathrm{X}$ & & 5 \\
\hline $\mathrm{q}_{\mathrm{t}-7}$ & & & & & & & $\mathrm{X}$ & & & & & & & & & & & & & & & & & 1 \\
\hline $\mathrm{q}_{\mathrm{t}-8}$ & & $\mathrm{X}$ & & & $\mathrm{X}$ & & & & & & & & & & & & & & & & & $\mathrm{X}$ & $\mathrm{X}$ & 4 \\
\hline $\mathrm{q}_{\mathrm{t}-\mathrm{9}}$ & & & & & & & & & & & & & & & & $\mathrm{X}$ & $\mathrm{X}$ & & & & & & & 2 \\
\hline $\mathrm{q}_{\mathrm{t}-10}$ & $\mathrm{X}$ & & & $\mathrm{X}$ & & & $\mathrm{X}$ & & & & & & & & & & & & & & & & & 3 \\
\hline MME12 & $\mathrm{X}$ & & & & $\mathrm{X}$ & & & & & $\mathrm{X}$ & & & & & & & & & & & & $\mathrm{X}$ & & 4 \\
\hline MME26 & & & & & & & & & & & & & & $\mathrm{X}$ & & & & & & & & & & 1 \\
\hline MACD & & & & & & & & & & $\mathrm{X}$ & & & & & $X$ & $\mathrm{X}$ & & & & & & & & 3 \\
\hline MME50 & & & & & & $\mathrm{X}$ & & & & & & $\mathrm{X}$ & & & $\mathrm{X}$ & & & & & & & & & 3 \\
\hline ind_MM & & & $\mathrm{X}$ & $\mathrm{X}$ & & & & & & & & & & & $\mathrm{X}$ & & & & & & & & & 3 \\
\hline $\mathrm{r} 2$ & & & & & & $\mathrm{X}$ & & & & & $\mathrm{X}$ & & & & & & & & & & & & & 2 \\
\hline $\mathrm{r} 5$ & & & & & & & & & & & $\mathrm{X}$ & $\mathrm{X}$ & & & & & $\mathrm{X}$ & $\mathrm{X}$ & & & & & $\mathrm{X}$ & 5 \\
\hline r39 & & & & & $\mathrm{X}$ & & $\mathrm{X}$ & & & & & & & $\mathrm{X}$ & & & & & & $\mathrm{X}$ & & & & 4 \\
\hline time & $\mathrm{X}$ & $\mathrm{X}$ & & & $\mathrm{X}$ & & & & & & $\mathrm{X}$ & & & & $\mathrm{X}$ & & & $\mathrm{X}$ & & & & & $\mathrm{X}$ & 7 \\
\hline Regimes & 5 & 11 & 8 & 5 & 11 & 4 & 11 & 9 & 5 & 11 & 11 & 11 & 2 & 6 & 11 & 11 & 9 & 4 & 11 & 4 & 5 & 11 & 8 & \\
\hline
\end{tabular}

\section{Statistical Evaluation}

Table 6 describes the statistical measures used for comparison of the STARX-Tree model (S-Tree), the naive approach (Naive), the random walk model (RW), the neural networks (NN) and the ARX model (ARX). The results are presented in Table 7. 
Table 6

Statistical Measures

\begin{tabular}{|l|l|}
\hline Measure & Description \\
\hline Mean absolute error & MAE $=\frac{1}{T} \sum_{t=1}^{T}\left|\tilde{y}_{t}-y_{t}\right|$ \\
\hline Root of mean square error & RMSE $=\sqrt{\frac{1}{T} \sum_{t=1}^{T}\left(\tilde{y}_{t}-y_{t}\right)^{2}}$ \\
\hline Correct directional changes & $\mathrm{CDC}=\frac{100}{T} \sum_{t=1}^{T} D_{t}$ \\
& $D_{t}=1$ se $\tilde{y}_{t} \times y_{t}>0 ;$ c.c. $D_{t}=0$ \\
\hline
\end{tabular}

Table 7

Statistical Evaluation In-Sample and Out-of-Sample

\begin{tabular}{|c|c|c|c|c|c|c|c|c|c|c|c|}
\hline \multirow{2}{*}{ Asset } & & \multicolumn{5}{|c|}{ In-sample } & \multicolumn{5}{|c|}{ Out-of-sample } \\
\hline & & RW & Naive & $\mathrm{NN}$ & ARX & S-Tree & RW & Naive & $\mathrm{NN}$ & ARX & S-Tree \\
\hline \multirow[t]{3}{*}{ AA } & MAE & 1.74 & 2.45 & 56.12 & 1.72 & 1.70 & 1.24 & 1.73 & 13.18 & 1.26 & 1.24 \\
\hline & RMSE & 2.33 & 3.27 & 87.45 & 2.32 & 2.29 & 1.63 & 2.25 & 17.57 & 1.65 & 1.63 \\
\hline & $\mathrm{CDC}$ & 47.68 & 48.00 & 48.74 & 52.37 & 52.09 & 49.45 & 54.23 & 51.84 & 50.18 & 51.84 \\
\hline \multirow[t]{3}{*}{ AIG } & MAE & 1.51 & 2.14 & 70.04 & 1.51 & 1.44 & 0.95 & 1.40 & 63.40 & 0.96 & 0.97 \\
\hline & RMSE & 2.51 & 3.47 & 83.26 & 2.49 & 2.00 & 1.39 & 1.95 & 83.66 & 1.41 & 1.42 \\
\hline & $\mathrm{CDC}$ & 48.87 & 50.90 & 48.37 & 51.77 & 51.22 & 45.59 & 48.35 & 50.00 & 49.45 & 46.32 \\
\hline \multirow[t]{3}{*}{ AXP } & MAE & 1.74 & 2.46 & 47.57 & 1.73 & 1.70 & 0.75 & 1.08 & 31.42 & 0.97 & 0.82 \\
\hline & RMSE & 2.33 & 3.29 & 71.19 & 2.34 & 2.31 & 1.12 & 1.55 & 34.09 & 1.32 & 1.20 \\
\hline & $\mathrm{CDC}$ & 49.06 & 47.40 & 48.51 & 52.60 & 55.49 & 49.26 & 48.16 & 50.37 & 49.82 & 48.90 \\
\hline \multirow[t]{3}{*}{$\mathrm{BA}$} & MAE & 1.63 & 2.32 & 68.54 & 1.61 & 1.61 & 1.05 & 1.61 & 31.36 & 1.06 & 1.06 \\
\hline & RMSE & 2.27 & 3.17 & 86.93 & 2.26 & 2.25 & 1.31 & 2.01 & 38.57 & 1.32 & 1.34 \\
\hline & $\mathrm{CDC}$ & 49.38 & 47.86 & 49.43 & 51.95 & 51.54 & 51.47 & 41.54 & 51.29 & 50.18 & 49.63 \\
\hline \multirow[t]{3}{*}{ CAT } & MAE & 1.64 & 2.31 & 63.77 & 1.63 & 1.58 & 1.14 & 1.61 & 140.34 & 1.15 & 1.15 \\
\hline & RMSE & 2.18 & 3.09 & 91.45 & 2.18 & 2.13 & 1.52 & 2.10 & 176.63 & 1.55 & 1.53 \\
\hline & $\mathrm{CDC}$ & 47.59 & 48.09 & 46.95 & 50.07 & 54.07 & 54.04 & 50.18 & 52.94 & 53.49 & 54.78 \\
\hline \multirow[t]{3}{*}{ DD } & MAE & 1.50 & 2.11 & 59.65 & 1.48 & 1.48 & 0.83 & 1.19 & 53.56 & 0.90 & 0.84 \\
\hline & RMSE & 2.00 & 2.81 & 89.92 & 1.99 & 1.98 & 1.09 & 1.54 & 76.09 & 1.18 & 1.11 \\
\hline & $\mathrm{CDC}$ & 47.13 & 47.73 & 47.63 & 52.87 & 52.37 & 49.45 & 48.35 & 49.82 & 49.45 & 48.53 \\
\hline \multirow[t]{3}{*}{ DIS } & MAE & 1.64 & 2.33 & 51.36 & 1.63 & 1.62 & 0.95 & 1.36 & 21.88 & 0.96 & 0.96 \\
\hline & RMSE & 2.28 & 3.21 & 64.70 & 2.27 & 2.24 & 1.35 & 1.91 & 30.61 & 1.36 & 1.35 \\
\hline & $\mathrm{CDC}$ & 47.59 & 47.36 & 48.32 & 51.26 & 50.99 & 48.35 & 47.98 & 46.51 & 48.35 & 46.51 \\
\hline \multirow[t]{3}{*}{ GE } & MAE & 1.46 & 2.07 & 52.99 & 1.45 & 1.40 & 0.74 & 1.04 & 19.59 & 0.76 & 0.82 \\
\hline & RMSE & 1.97 & 2.78 & 89.77 & 1.95 & 1.90 & 0.95 & 1.32 & 24.21 & 0.97 & 1.04 \\
\hline & $\mathrm{CDC}$ & 48.60 & 48.46 & 49.01 & 52.78 & 54.66 & 47.06 & 49.26 & 51.29 & 49.26 & 47.06 \\
\hline \multirow[t]{3}{*}{ GM } & MAE & 1.59 & 2.31 & 75.35 & 1.56 & 1.55 & 1.34 & 1.91 & 77.54 & 1.36 & 1.38 \\
\hline & RMSE & 2.15 & 3.12 & 98.66 & 2.13 & 2.10 & 2.07 & 2.86 & 110.90 & 2.09 & 2.16 \\
\hline & $\mathrm{CDC}$ & 47.31 & 46.03 & 47.04 & 52.96 & 54.02 & 53.68 & 50.18 & 45.22 & 50.55 & 49.45 \\
\hline
\end{tabular}


(Table 7 continued)

\begin{tabular}{|c|c|c|c|c|c|c|c|c|c|c|c|}
\hline \multirow{2}{*}{ Asset } & & \multicolumn{5}{|c|}{ In-sample } & \multicolumn{5}{|c|}{ Out-of-sample } \\
\hline & & RW & Naive & $\mathrm{NN}$ & Asset & S-Tree & $\mathrm{RW}$ & Naive & $\mathrm{NN}$ & Asset & S-Tree \\
\hline \multirow[t]{3}{*}{ HD } & MAE & 1.81 & 2.52 & 66.48 & 1.80 & 1.79 & 0.95 & 1.38 & 32.46 & 1.02 & 1.80 \\
\hline & RMSE & 2.76 & 3.81 & 80.41 & 2.74 & 2.56 & 1.22 & 1.74 & 47.43 & 1.30 & 10.12 \\
\hline & $\mathrm{CDC}$ & 48.28 & 48.14 & 50.16 & 52.18 & 50.34 & 50.37 & 43.93 & 51.29 & 50.37 & 50.00 \\
\hline \multirow[t]{3}{*}{ HOM } & MAE & 1.75 & 2.47 & 55.13 & 1.75 & 1.67 & 0.99 & 1.42 & 25.75 & 1.12 & 1.03 \\
\hline & RMSE & 2.49 & 3.45 & 79.04 & 2.50 & 2.39 & 1.30 & 1.85 & 32.93 & 1.42 & 1.35 \\
\hline & $\mathrm{CDC}$ & 48.74 & 46.39 & 49.89 & 51.17 & 54.98 & 50.18 & 48.35 & 49.26 & 49.82 & 47.06 \\
\hline \multirow[t]{3}{*}{ IBM } & MAE & 1.66 & 2.43 & 51.96 & 1.64 & 1.60 & 0.75 & 1.06 & 24.08 & 0.76 & 0.76 \\
\hline & RMSE & 2.35 & 3.39 & 81.74 & 2.33 & 2.22 & 1.02 & 1.41 & 38.49 & 1.03 & 1.03 \\
\hline & $\mathrm{CDC}$ & 49.98 & 47.08 & 50.34 & 53.01 & 53.97 & 48.16 & 48.71 & 50.37 & 50.00 & 48.16 \\
\hline \multirow[t]{3}{*}{ JNJ } & MAE & 1.25 & 1.74 & 77.10 & 1.22 & 1.24 & 0.67 & 0.98 & 98.07 & 0.68 & 0.68 \\
\hline & RMSE & 1.68 & 2.33 & 88.14 & 1.65 & 1.67 & 0.90 & 1.29 & 112.99 & 0.91 & 0.91 \\
\hline & $\mathrm{CDC}$ & 50.30 & 47.73 & 50.30 & 53.65 & 52.09 & 47.06 & 46.14 & 47.06 & 51.10 & 47.43 \\
\hline \multirow[t]{3}{*}{ JPM } & MAE & 1.71 & 2.49 & 47.58 & 1.70 & 1.70 & 0.74 & 1.04 & 31.35 & 0.74 & 0.75 \\
\hline & RMSE & 2.39 & 3.44 & 81.39 & 2.39 & 2.39 & 0.96 & 1.34 & 36.30 & 0.96 & 0.97 \\
\hline & $\mathrm{CDC}$ & 48.60 & 46.12 & 47.40 & 52.18 & 51.63 & 48.71 & 49.63 & 48.35 & 49.08 & 48.53 \\
\hline \multirow[t]{3}{*}{$\mathrm{KO}$} & MAE & 1.33 & 1.87 & 49.56 & 1.31 & 1.28 & 0.65 & 0.94 & 18.24 & 0.68 & 0.67 \\
\hline & RMSE & 1.79 & 2.49 & 86.00 & 1.77 & 1.73 & 0.93 & 1.28 & 22.79 & 0.95 & 0.94 \\
\hline & $\mathrm{CDC}$ & 48.00 & 46.26 & 47.31 & 52.23 & 53.47 & 48.16 & 48.90 & 49.82 & 48.16 & 49.26 \\
\hline \multirow[t]{3}{*}{ MCD } & MAE & 1.38 & 1.94 & 55.47 & 1.36 & 1.33 & 1.02 & 1.49 & 24.80 & 1.11 & 1.04 \\
\hline & RMSE & 1.90 & 2.65 & 91.40 & 1.89 & 1.86 & 1.35 & 1.97 & 40.99 & 1.44 & 1.38 \\
\hline & CDC & 47.68 & 45.43 & 47.59 & 51.81 & 54.48 & 51.65 & 48.53 & 49.08 & 47.24 & 50.55 \\
\hline \multirow[t]{3}{*}{ MMM } & MAE & 1.24 & 1.82 & 63.16 & 1.23 & 1.21 & 0.82 & 1.21 & 93.08 & 0.83 & 0.87 \\
\hline & RMSE & 1.69 & 2.40 & 88.95 & 1.68 & 1.67 & 1.14 & 1.63 & 115.82 & 1.15 & 1.21 \\
\hline & $\mathrm{CDC}$ & 47.96 & 45.98 & 48.19 & 52.92 & 55.03 & 50.37 & 47.43 & 50.37 & 47.24 & 47.61 \\
\hline \multirow[t]{3}{*}{ MO } & MAE & 1.52 & 2.19 & 37.76 & 1.51 & 1.50 & 0.83 & 1.17 & 17.72 & 0.84 & 0.84 \\
\hline & RMSE & 2.19 & 3.09 & 52.62 & 2.18 & 2.16 & 1.26 & 1.74 & 21.42 & 1.27 & 1.27 \\
\hline & $\mathrm{CDC}$ & 50.53 & 47.82 & 51.03 & 52.64 & 54.16 & 53.68 & 50.74 & 50.18 & 52.02 & 51.10 \\
\hline \multirow[t]{3}{*}{ MRK } & MAE & 1.41 & 1.95 & 50.74 & 1.39 & 1.40 & 1.06 & 1.56 & 34.42 & 1.09 & 1.16 \\
\hline & RMSE & 1.87 & 2.57 & 80.05 & 1.85 & 1.85 & 2.06 & 2.92 & 42.02 & 2.09 & 2.17 \\
\hline & $\mathrm{CDC}$ & 49.56 & 49.33 & 50.99 & 55.35 & 52.78 & 49.63 & 47.43 & 48.90 & 47.79 & 48.35 \\
\hline \multirow[t]{3}{*}{ PFE } & MAE & 1.57 & 2.20 & 70.12 & 1.54 & 1.53 & 1.00 & 1.38 & 29.12 & 6.74 & 1.04 \\
\hline & RMSE & 2.05 & 2.85 & 92.29 & 2.02 & 2.00 & 1.44 & 1.90 & 44.00 & 6.97 & 1.50 \\
\hline & $\mathrm{CDC}$ & 49.84 & 48.23 & 50.62 & 55.08 & 55.44 & 45.77 & 50.74 & 47.43 & 53.31 & 48.53 \\
\hline \multirow[t]{3}{*}{ PG } & MAE & 1.28 & 1.83 & 61.04 & 1.26 & 1.26 & 0.68 & 1.00 & 40.95 & 0.71 & 0.69 \\
\hline & RMSE & 1.91 & 2.71 & 70.96 & 1.89 & 1.88 & 0.88 & 1.29 & 43.58 & 0.91 & 0.89 \\
\hline & $\mathrm{CDC}$ & 50.25 & 47.68 & 50.85 & 53.79 & 52.18 & 51.29 & 46.51 & 51.29 & 47.43 & 54.41 \\
\hline \multirow[t]{3}{*}{ UTX } & MAE & 1.47 & 2.09 & 51.63 & 1.46 & 1.44 & 0.82 & 1.18 & 29.35 & 17.88 & 0.83 \\
\hline & RMSE & 2.07 & 2.96 & 70.29 & 2.07 & 2.04 & 1.05 & 1.49 & 36.74 & 17.95 & 1.06 \\
\hline & $\mathrm{CDC}$ & 49.98 & 46.62 & 50.76 & 51.86 & 54.07 & 50.74 & 48.16 & 48.53 & 48.90 & 51.47 \\
\hline
\end{tabular}


(Table 7 continued)

\begin{tabular}{|c|c|c|c|c|c|c|c|c|c|c|c|}
\hline \multirow{2}{*}{ Asset } & & \multicolumn{5}{|c|}{ In-sample } & \multicolumn{5}{|c|}{ Out-of-sample } \\
\hline & & RW & Naive & $\mathrm{NN}$ & Asset & S-Tree & RW & Naive & $\mathrm{NN}$ & Asset & S-Tree \\
\hline \multirow[t]{3}{*}{ WMT } & MAE & 1.60 & 2.30 & 60.32 & 1.60 & 1.56 & 0.78 & 1.08 & 44.68 & 10.18 & 0.80 \\
\hline & RMSE & 2.14 & 3.01 & 88.71 & 2.14 & 2.11 & 1.02 & 1.39 & 55.06 & 10.25 & 1.04 \\
\hline & $\mathrm{CDC}$ & 47.91 & 43.18 & 47.63 & 50.99 & 51.95 & 47.43 & 48.71 & 47.43 & 51.84 & 52.57 \\
\hline
\end{tabular}

According to Table 7, the STARX-Tree methodology outperformed the other models when the measures were evaluated in-sample, however, for out-of-sample data, the random walk model gave better results in most of the series.

\section{Financial Evaluation}

Financial evaluation is the main goal of this comparison because the forecasting methods are used to develop automatic trading strategies. To see if they were successful, we measure the financial indicators in Table 8 for in-sample and out-of-sample data.

Table 8

\section{Financial Measures}

\begin{tabular}{|l|l|}
\hline Measure & Description \\
\hline Annualized returns & $\begin{array}{l}R^{A}=252 \times \frac{1}{T} \sum_{i=1}^{N T} R_{i} \\
\mathrm{NT}: \text { num. transactions }\end{array}$ \\
\hline Accumulated returns & $R^{C}=\sum_{i=1}^{N T} R_{i}$ \\
\hline Annualized volatility & $\sigma^{A}=\sqrt{252} \times \sqrt{\frac{1}{T-1} \sum_{i=1}^{N T}\left(R_{i}-\overline{\mathrm{R}}\right)^{2}}$ \\
\hline Sharpe ratio & $\mathrm{SR}=\frac{R^{A}}{\sigma^{A}}$ \\
\hline$\%$ Winning trades & $\begin{array}{l}\sum_{i=1}^{N T} P F_{i} \\
P T\end{array}$ \\
\hline
\end{tabular}

The measures of highest interest are the annualized and accumulated returns, and the annualized volatility, from which we compute the Sharpe ratio. The results are presented in Table 8. Using this criterion, the financial results in Table 9 for in-sample data showed that STARX-Tree methodology outperformed the other methods in 15 series. However, for out-of-sample data, the results were more balanced.

An interesting way to evaluate the financial performance is the aggregate analysis of the 23 assets, evaluating the average returns. In order to have a more realistic idea of the gains of the automatic trading, we included transaction costs and a comparison to the Buy and Hold strategy which gives in fact the accumulated return of the market.

In the literature, authors tend to use a transaction cost in the range of $0.01 \%-0.05 \%$ per trade (e.g., Neely, Weller, \& Dittmar, 1997); LeBaron (1999); Qi and Wu (2002); Neely and Weller (2003). Lyons (2001) argues that a transaction cost (for a one-way trade) of 1-2 basis points for institutional traders is the norm, while Neely, Weller and Dittmar (1997) and Neely and Weller (2003) suggest a transaction cost of 2-2.5 basis points. Dunis and Williams (2002) argue that a cost of 3 basis points (one way) between market makers was more realistic in the 1990s. We consider the transaction cost of 3 basis points (bp) per trade. 
Table 9

Financial Evaluation In-Sample and Out-of-Sample

\begin{tabular}{|c|c|c|c|c|c|c|c|c|c|c|c|}
\hline \multirow{2}{*}{\multicolumn{2}{|c|}{ Asset(\%) }} & \multicolumn{5}{|c|}{ In-sample } & \multicolumn{5}{|c|}{ Out-of-sample } \\
\hline & & \multirow{2}{*}{$\frac{\mathrm{RW}}{12.99}$} & \multirow{2}{*}{$\begin{array}{r}\text { Naive } \\
7.67\end{array}$} & \multirow{2}{*}{$\begin{array}{l}\mathrm{NN} \\
18.81\end{array}$} & \multirow{2}{*}{$\begin{aligned} \text { ARX } \\
29.21\end{aligned}$} & \multirow{2}{*}{$\begin{array}{r}\text { S-Tree } \\
33.38\end{array}$} & \multirow{2}{*}{$\frac{\mathrm{RW}}{-3.53}$} & \multirow{2}{*}{$\begin{array}{r}\text { Naive } \\
21.89\end{array}$} & \multirow{2}{*}{$\begin{array}{r}\mathrm{NN} \\
8.28\end{array}$} & \multirow{2}{*}{$\begin{array}{r}\text { ARX } \\
-5.07\end{array}$} & \multirow{2}{*}{$\frac{\text { S-Tree }}{13.44}$} \\
\hline AA & Annual. Ret & & & & & & & & & & \\
\hline & Cum. Ret & 112.22 & 66.28 & 162.54 & 252.35 & 288.36 & -7.62 & 47.26 & 17.88 & -10.95 & 29.02 \\
\hline & Annual. Vol & 32.69 & 24.58 & 31.86 & 26.61 & 27.39 & 0.00 & 18.83 & 19.71 & 24.13 & 22.34 \\
\hline & Sharpe Ratio & 0.40 & 0.31 & 0.59 & 1.10 & 1.22 & $-\operatorname{Inf}$ & 1.16 & 0.42 & -0.21 & 0.60 \\
\hline & Num. Trade & 3.00 & 542.00 & 209.00 & 437.00 & 491.00 & 1.00 & 121.00 & 160.00 & 50.00 & 113.00 \\
\hline & Win. Trade & 0.33 & 0.35 & 0.51 & 0.54 & 0.57 & 0.00 & 0.45 & 0.53 & 0.50 & 0.52 \\
\hline \multirow[t]{6}{*}{ AIG } & Annual. Ret & -8.50 & 16.27 & -4.20 & 18.41 & 23.96 & -10.87 & 6.34 & 5.07 & -7.71 & -3.27 \\
\hline & Cum. Ret & -73.47 & 140.52 & -36.26 & 159.08 & 207.01 & -23.46 & 13.68 & 10.94 & -16.65 & -7.05 \\
\hline & Annual. Vol & 10.76 & 24.80 & 24.08 & 23.22 & 25.68 & 2.57 & 12.70 & 21.91 & 11.55 & 21.81 \\
\hline & Sharpe Ratio & -0.79 & 0.66 & -0.17 & 0.79 & 0.93 & -4.23 & 0.50 & 0.23 & -0.67 & -0.15 \\
\hline & Num. Trade & 20.00 & 519.00 & 62.00 & 320.00 & 272.00 & 13.00 & 138.00 & 44.00 & 57.00 & 53.00 \\
\hline & Win. Trade & 0.00 & 0.39 & 0.48 & 0.61 & 0.61 & 0.00 & 0.34 & 0.59 & 0.46 & 0.55 \\
\hline \multirow[t]{6}{*}{ AXP } & Annual. Ret & 16.05 & 12.01 & 13.07 & 19.63 & 36.55 & 3.75 & 0.78 & -0.01 & 0.81 & 5.79 \\
\hline & Cum. Ret & 138.64 & 103.77 & 112.91 & 169.57 & 315.72 & 8.09 & 1.68 & -0.02 & 1.75 & 12.50 \\
\hline & Annual. Vol & 39.63 & 23.82 & 25.58 & 24.64 & 26.47 & 0.00 & 10.93 & 10.97 & 1.98 & 14.83 \\
\hline & Sharpe Ratio & 0.40 & 0.50 & 0.51 & 0.80 & 1.38 & Inf & 0.07 & 0.00 & 0.41 & 0.39 \\
\hline & Num. Trade & 3.00 & 545.00 & 509.00 & 288.00 & 271.00 & 1.00 & 136.00 & 101.00 & 2.00 & 122.00 \\
\hline & Win. Trade & 0.33 & 0.37 & 0.52 & 0.65 & 0.69 & 1.00 & 0.32 & 0.52 & 0.50 & 0.57 \\
\hline \multirow[t]{6}{*}{$\mathrm{BA}$} & Annual. Ret & 5.56 & 7.66 & 10.78 & 15.31 & 15.35 & 28.21 & -9.26 & 20.32 & 23.06 & 20.05 \\
\hline & Cum. Ret & 48.00 & 66.22 & 93.14 & 132.29 & 132.62 & 60.91 & -19.99 & 43.87 & 49.79 & 43.29 \\
\hline & Annual. Vol & 0.00 & 22.65 & 37.40 & 29.29 & 31.78 & 0.00 & 14.55 & 14.91 & 22.42 & 17.46 \\
\hline & Sharpe Ratio & Inf & 0.34 & 0.29 & 0.52 & 0.48 & Inf & -0.64 & 1.36 & 1.03 & 1.15 \\
\hline & Num. Trade & 1.00 & 536.00 & 336.00 & 430.00 & 316.00 & 1.00 & 158.00 & 109.00 & 27.00 & 101.00 \\
\hline & Win. Trade & 1.00 & 0.38 & 0.49 & 0.60 & 0.57 & 1.00 & 0.31 & 0.54 & 0.56 & 0.53 \\
\hline \multirow[t]{6}{*}{ CAT } & Annual. Ret & 12.77 & 9.74 & 6.04 & 17.07 & 35.20 & 19.30 & 17.78 & 17.26 & 15.88 & 24.38 \\
\hline & Cum. Ret & 110.35 & 84.10 & 52.22 & 147.44 & 304.05 & 41.67 & 38.38 & 37.26 & 34.29 & 52.63 \\
\hline & Annual. Vol & 0.00 & 24.96 & 29.68 & 25.30 & 24.48 & 0.00 & 18.78 & 19.11 & 19.30 & 19.18 \\
\hline & Sharpe Ratio & Inf & 0.39 & 0.20 & 0.67 & 1.44 & Inf & 0.95 & 0.90 & 0.82 & 1.27 \\
\hline & Num. Trade & 1.00 & 531.00 & 368.00 & 524.00 & 510.00 & 1.00 & 134.00 & 11.00 & 20.00 & 79.00 \\
\hline & Win. Trade & 1.00 & 0.35 & 0.52 & 0.52 & 0.56 & 1.00 & 0.43 & 0.64 & 0.80 & 0.58 \\
\hline \multirow[t]{6}{*}{ DD } & Annual. Ret & 4.15 & 7.17 & 2.63 & 17.49 & 19.98 & 3.47 & -2.12 & 1.51 & 2.84 & 0.26 \\
\hline & Cum. Ret & 35.87 & 61.95 & 22.74 & 151.05 & 172.63 & 7.48 & -4.57 & 3.26 & 6.13 & 0.56 \\
\hline & Annual. Vol & 0.00 & 19.23 & 28.23 & 23.39 & 22.70 & 0.00 & 12.58 & 19.96 & 11.51 & 14.40 \\
\hline & Sharpe Ratio & Inf & 0.37 & 0.09 & 0.75 & 0.88 & Inf & -0.17 & 0.08 & 0.25 & 0.02 \\
\hline & Num. Trade & 1.00 & 540.00 & 195.00 & 456.00 & 535.00 & 1.00 & 138.00 & 83.00 & 124.00 & 156.00 \\
\hline & Win. Trade & 1.00 & 0.38 & 0.46 & 0.58 & 0.52 & 1.00 & 0.30 & 0.45 & 0.50 & 0.47 \\
\hline \multirow[t]{3}{*}{ DIS } & Annual. Ret & -3.30 & 8.47 & 1.77 & 25.25 & 19.77 & 2.80 & 1.47 & -0.82 & 4.00 & 3.59 \\
\hline & Cum. Ret & -28.51 & 73.20 & 15.29 & 218.16 & 170.83 & 6.05 & 3.17 & -1.77 & 8.63 & 7.75 \\
\hline & Annual. Vol & 8.46 & 22.64 & 31.03 & 26.26 & 28.99 & 0.00 & 16.71 & 20.20 & 18.64 & 16.50 \\
\hline
\end{tabular}


(Table 9 continued)

\begin{tabular}{|c|c|c|c|c|c|c|c|c|c|c|c|}
\hline \multirow{2}{*}{ Asset(\%) } & & \multicolumn{5}{|c|}{ In-sample } & \multicolumn{5}{|c|}{ Out-of-sample } \\
\hline & & RW & Naive & $\mathrm{NN}$ & Asset & $(\%)$ & RW & Naive & $\mathrm{NN}$ & Asset & $(\%)$ \\
\hline & Sharpe Ratio & -0.39 & 0.37 & 0.06 & 0.96 & 0.68 & Inf & 0.09 & -0.04 & 0.21 & 0.22 \\
\hline & Num. Trade & 22.00 & 531.00 & 346.00 & 507.00 & 302.00 & 1.00 & 135.00 & 109.00 & 122.00 & 79.00 \\
\hline & Win. Trade & 0.05 & 0.37 & 0.47 & 0.54 & 0.58 & 1.00 & 0.30 & 0.45 & 0.47 & 0.51 \\
\hline \multirow[t]{6}{*}{ GE } & Annual. Ret & 12.51 & 12.24 & 16.23 & 31.35 & 39.16 & 9.60 & 5.03 & 6.60 & 3.86 & 6.66 \\
\hline & Cum. Ret & 108.06 & 105.75 & 140.20 & 270.85 & 338.27 & 20.73 & 10.87 & 14.25 & 8.33 & 14.38 \\
\hline & Annual. Vol & 36.15 & 21.29 & 24.59 & 23.83 & 27.70 & 0.00 & 9.46 & 10.73 & 11.57 & 12.21 \\
\hline & Sharpe Ratio & 0.35 & 0.57 & 0.66 & 1.32 & 1.41 & Inf & 0.53 & 0.62 & 0.33 & 0.55 \\
\hline & Num. Trade & 7.00 & 537.00 & 368.00 & 400.00 & 474.00 & 1.00 & 136.00 & 197.00 & 117.00 & 52.00 \\
\hline & Win. Trade & 0.14 & 0.37 & 0.49 & 0.62 & 0.60 & 1.00 & 0.33 & 0.49 & 0.49 & 0.56 \\
\hline \multirow[t]{6}{*}{ GM } & Annual. Ret & -4.40 & -0.42 & -0.52 & 21.41 & 25.85 & -3.68 & -3.23 & -38.77 & -10.44 & -19.11 \\
\hline & Cum. Ret & -37.98 & -3.65 & -4.45 & 184.95 & 223.31 & -7.94 & -6.97 & -83.68 & -22.55 & -41.24 \\
\hline & Annual. Vol & 2.62 & 20.60 & 17.22 & 22.85 & 24.42 & 0.91 & 17.81 & 38.39 & 21.71 & 18.31 \\
\hline & Sharpe Ratio & -1.68 & -0.02 & -0.03 & 0.94 & 1.06 & -4.04 & -0.18 & -1.01 & -0.48 & -1.04 \\
\hline & Num. Trade & 13.00 & 558.00 & 48.00 & 540.00 & 528.00 & 5.00 & 135.00 & 7.00 & 104.00 & 132.00 \\
\hline & Win. Trade & 0.08 & 0.35 & 0.56 & 0.56 & 0.57 & 0.00 & 0.29 & 0.43 & 0.48 & 0.42 \\
\hline \multirow[t]{6}{*}{ HD } & Annual. Ret & -0.21 & 15.23 & 6.72 & 31.01 & 18.25 & 4.10 & -7.08 & 5.13 & 5.76 & 6.97 \\
\hline & Cum. Ret & -1.85 & 131.58 & 58.02 & 267.92 & 157.68 & 8.85 & -15.28 & 11.07 & 12.44 & 15.05 \\
\hline & Annual. Vol & 15.04 & 29.18 & 39.77 & 30.39 & 35.58 & 0.00 & 14.52 & 14.69 & 15.68 & 13.97 \\
\hline & Sharpe Ratio & -0.01 & 0.52 & 0.17 & 1.02 & 0.51 & $\operatorname{Inf}$ & -0.49 & 0.35 & 0.37 & 0.50 \\
\hline & Num. Trade & 13.00 & 535.00 & 434.00 & 353.00 & 124.00 & 1.00 & 149.00 & 110.00 & 109.00 & 50.00 \\
\hline & Win. Trade & 0.08 & 0.38 & 0.55 & 0.55 & 0.58 & 1.00 & 0.31 & 0.49 & 0.59 & 0.52 \\
\hline \multirow[t]{6}{*}{ HOM } & Annual. Ret & 4.57 & 12.31 & 4.89 & 18.55 & 38.52 & 9.40 & 0.18 & -0.84 & 7.92 & -7.43 \\
\hline & Cum. Ret & 39.44 & 106.32 & 42.29 & 160.26 & 332.77 & 20.29 & 0.39 & -1.81 & 17.11 & -16.05 \\
\hline & Annual. Vol & 12.56 & 27.11 & 29.70 & 32.62 & 35.02 & 0.00 & 14.62 & 9.27 & 2.00 & 13.02 \\
\hline & Sharpe Ratio & 0.36 & 0.45 & 0.16 & 0.57 & 1.10 & Inf & 0.01 & -0.09 & 3.97 & -0.57 \\
\hline & Num. Trade & 4.00 & 546.00 & 466.00 & 434.00 & 520.00 & 1.00 & 138.00 & 112.00 & 3.00 & 138.00 \\
\hline & $\begin{array}{l}\text { Win. Trade } \\
\text { continue in } \\
\text { nextpage }\end{array}$ & 0.25 & 0.34 & 0.51 & 0.56 & 0.59 & 1.00 & 0.33 & 0.48 & 1.00 & 0.47 \\
\hline \multirow[t]{6}{*}{ IBM } & Annual. Ret & 16.96 & 5.55 & 18.96 & 29.92 & 44.49 & -3.57 & 1.85 & -0.05 & 1.90 & -1.81 \\
\hline & Cum. Ret & 146.53 & 47.95 & 163.75 & 258.48 & 384.33 & -7.71 & 4.00 & -0.11 & 4.10 & -3.91 \\
\hline & Annual. Vol & 42.42 & 26.43 & 27.87 & 28.90 & 31.95 & 0.00 & 11.63 & 7.10 & 8.32 & 14.27 \\
\hline & Sharpe Ratio & 0.40 & 0.21 & 0.68 & 1.04 & 1.39 & $-\operatorname{Inf}$ & 0.16 & -0.01 & 0.23 & -0.13 \\
\hline & Num. Trade & 3.00 & 564.00 & 405.00 & 272.00 & 280.00 & 1.00 & 139.00 & 111.00 & 38.00 & 25.00 \\
\hline & Win. Trade & 0.33 & 0.35 & 0.51 & 0.61 & 0.55 & 0.00 & 0.31 & 0.49 & 0.32 & 0.44 \\
\hline \multirow[t]{4}{*}{ JNJ } & Annual. Ret & 13.57 & 9.52 & 14.05 & 31.77 & 19.82 & 9.41 & -2.56 & 9.41 & 8.22 & 10.94 \\
\hline & Cum. Ret & 117.20 & 82.25 & 121.40 & 274.46 & 171.25 & 20.32 & -5.53 & 20.32 & 17.74 & 23.62 \\
\hline & Annual. Vol & 29.29 & 18.24 & 19.57 & 18.95 & 18.93 & 0.00 & 8.63 & 0.00 & 7.14 & 13.17 \\
\hline & Sharpe Ratio & 0.46 & 0.52 & 0.72 & 1.68 & 1.05 & Inf & -0.30 & Inf & 1.15 & 0.83 \\
\hline \multirow[t]{2}{*}{ JPM } & Num. Trade & 2.00 & 547.00 & 2.00 & 478.00 & 457.00 & 1.00 & 144.00 & 1.00 & 32.00 & 58.00 \\
\hline & Win. Trade & 0.50 & 0.36 & 1.00 & 0.55 & 0.58 & 1.00 & 0.34 & 1.00 & 0.63 & 0.53 \\
\hline
\end{tabular}


(Table 9 continued)

\begin{tabular}{|c|c|c|c|c|c|c|c|c|c|c|c|}
\hline \multirow{2}{*}{\multicolumn{2}{|c|}{ Asset(\%) }} & \multicolumn{5}{|c|}{ In-sample } & \multicolumn{5}{|c|}{ Out-of-sample } \\
\hline & & RW & Naive & $\mathrm{NN}$ & Asset & $(\%)$ & RW & Naive & $\mathrm{NN}$ & Asset & $(\%)$ \\
\hline & Annual. Ret & 8.21 & 9.26 & 10.76 & 23.21 & 18.46 & 4.03 & 1.50 & 1.52 & 0.10 & 0.40 \\
\hline & Cum. Ret & 70.95 & 80.02 & 93.00 & 200.53 & 159.43 & 8.70 & 3.24 & 3.29 & 0.22 & 0.86 \\
\hline & Annual. Vol & 25.51 & 23.47 & 29.49 & 26.38 & 34.55 & 0.00 & 10.52 & 9.78 & 12.97 & 14.12 \\
\hline & Sharpe Ratio & 0.32 & 0.39 & 0.37 & 0.88 & 0.53 & Inf & 0.14 & 0.16 & 0.01 & 0.03 \\
\hline & Num. Trade & 5.00 & 556.00 & 714.00 & 391.00 & 363.00 & 1.00 & 132.00 & 210.00 & 119.00 & 84.00 \\
\hline & Win. Trade & 0.20 & 0.36 & 0.48 & 0.61 & 0.62 & 1.00 & 0.38 & 0.49 & 0.53 & 0.55 \\
\hline \multirow[t]{6}{*}{$\mathrm{KO}$} & Annual. Ret & 5.56 & 4.23 & -2.32 & 21.59 & 30.00 & -6.68 & -4.32 & -3.94 & -7.26 & 3.28 \\
\hline & Cum. Ret & 48.04 & 36.57 & -20.08 & 186.51 & 259.15 & -14.43 & -9.32 & -8.51 & -15.68 & 7.08 \\
\hline & Annual. Vol & 0.00 & 18.00 & 23.74 & 22.72 & 22.62 & 0.00 & 9.07 & 10.53 & 11.57 & 11.17 \\
\hline & Sharpe Ratio & Inf & 0.24 & -0.10 & 0.95 & 1.33 & $-\operatorname{Inf}$ & -0.48 & -0.37 & -0.63 & 0.29 \\
\hline & Num. Trade & 1.00 & 559.00 & 556.00 & 469.00 & 397.00 & 1.00 & 134.00 & 186.00 & 65.00 & 58.00 \\
\hline & Win. Trade & 1.00 & 0.34 & 0.49 & 0.55 & 0.57 & 0.00 & 0.36 & 0.47 & 0.48 & 0.52 \\
\hline \multirow[t]{6}{*}{$\mathrm{MCD}$} & Annual. Ret & 2.95 & 5.95 & 4.07 & 14.48 & 29.59 & 12.44 & -0.52 & 5.87 & 2.39 & 6.88 \\
\hline & Cum. Ret & 25.51 & 51.39 & 35.15 & 125.12 & 255.59 & 26.86 & -1.13 & 12.67 & 5.16 & 14.86 \\
\hline & Annual. Vol & 12.91 & 21.75 & 26.12 & 22.98 & 22.11 & 0.00 & 15.38 & 15.34 & 10.95 & 17.33 \\
\hline & Sharpe Ratio & 0.23 & 0.27 & 0.16 & 0.63 & 1.34 & Inf & -0.03 & 0.38 & 0.22 & 0.40 \\
\hline & Num. Trade & 5.00 & 548.00 & 189.00 & 459.00 & 555.00 & 1.00 & 138.00 & 170.00 & 120.00 & 111.00 \\
\hline & Win. Trade & 0.20 & 0.34 & 0.52 & 0.52 & 0.56 & 1.00 & 0.38 & 0.52 & 0.50 & 0.51 \\
\hline \multirow[t]{6}{*}{ MMM } & Annual. Ret & 11.38 & 6.17 & 12.20 & 21.22 & 31.29 & -0.72 & -7.37 & -0.72 & -4.84 & -4.49 \\
\hline & Cum. Ret & 98.28 & 53.27 & 105.43 & 183.29 & 270.34 & -1.55 & -15.91 & -1.55 & -10.44 & -9.69 \\
\hline & Annual. Vol & 32.39 & 18.51 & 17.59 & 16.91 & 17.26 & 0.00 & 11.42 & 0.00 & 12.08 & 13.01 \\
\hline & Sharpe Ratio & 0.35 & 0.33 & 0.69 & 1.25 & 1.81 & $-\operatorname{Inf}$ & -0.65 & $-\operatorname{Inf}$ & -0.40 & -0.35 \\
\hline & Num. Trade & 5.00 & 554.00 & 21.00 & 476.00 & 482.00 & 1.00 & 143.00 & 1.00 & 152.00 & 99.00 \\
\hline & Win. Trade & 0.20 & 0.34 & 0.67 & 0.62 & 0.62 & 0.00 & 0.31 & 0.00 & 0.49 & 0.55 \\
\hline \multirow[t]{6}{*}{ MO } & Annual. Ret & 7.39 & 5.87 & 14.87 & 23.90 & 36.08 & 22.19 & 20.42 & 10.44 & 26.39 & 22.57 \\
\hline & Cum. Ret & 63.83 & 50.71 & 128.50 & 206.49 & 311.70 & 47.90 & 44.07 & 22.53 & 56.97 & 48.72 \\
\hline & Annual. Vol & 23.15 & 23.43 & 32.10 & 27.67 & 26.32 & 0.00 & 15.21 & 18.29 & 16.28 & 17.29 \\
\hline & Sharpe Ratio & 0.32 & 0.25 & 0.46 & 0.86 & 1.37 & Inf & 1.34 & 0.57 & 1.62 & 1.31 \\
\hline & Num. Trade & 4.00 & 541.00 & 353.00 & 366.00 & 302.00 & 1.00 & 131.00 & 114.00 & 106.00 & 105.00 \\
\hline & Win. Trade & 0.25 & 0.39 & 0.54 & 0.61 & 0.63 & 1.00 & 0.44 & 0.57 & 0.60 & 0.53 \\
\hline \multirow[t]{6}{*}{ MRK } & Annual. Ret & 8.54 & 12.47 & 11.17 & 31.12 & 20.21 & -14.09 & -9.44 & 2.38 & -21.64 & -20.17 \\
\hline & Cum. Ret & 73.75 & 107.71 & 96.47 & 268.84 & 174.55 & -30.43 & -20.37 & 5.13 & -46.72 & -43.53 \\
\hline & Annual. Vol & 21.97 & 19.92 & 17.86 & 21.41 & 27.02 & 0.00 & 25.02 & 12.00 & 33.39 & 28.56 \\
\hline & Sharpe Ratio & 0.39 & 0.63 & 0.63 & 1.45 & 0.75 & $-\operatorname{Inf}$ & -0.38 & 0.20 & -0.65 & -0.71 \\
\hline & Num. Trade & 3.00 & 530.00 & 586.00 & 428.00 & 228.00 & 1.00 & 138.00 & 96.00 & 101.00 & 121.00 \\
\hline & Win. Trade & 0.33 & 0.39 & 0.55 & 0.56 & 0.57 & 0.00 & 0.35 & 0.49 & 0.51 & 0.50 \\
\hline \multirow[t]{5}{*}{ PFE } & Annual. Ret & 16.70 & 12.97 & 19.78 & 43.06 & 47.41 & -13.53 & 6.21 & -5.75 & -2.55 & -6.59 \\
\hline & Cum. Ret & 144.23 & 112.05 & 170.91 & 372.01 & 409.55 & -29.21 & 13.42 & -12.42 & -5.51 & -14.23 \\
\hline & Annual. Vol & 41.71 & 22.49 & 44.04 & 22.42 & 23.34 & 0.00 & 14.90 & 18.28 & 21.11 & 16.00 \\
\hline & Sharpe Ratio & 0.40 & 0.58 & 0.45 & 1.92 & 2.03 & $-\operatorname{Inf}$ & 0.42 & -0.31 & -0.12 & -0.41 \\
\hline & Num. Trade & 3.00 & 536.00 & 273.00 & 395.00 & 421.00 & 1.00 & 131.00 & 109.00 & 108.00 & 99.00 \\
\hline
\end{tabular}


(Table 9 continued)

\begin{tabular}{|c|c|c|c|c|c|c|c|c|c|c|c|}
\hline \multirow{2}{*}{ Asset(\%) } & & \multicolumn{5}{|c|}{ In-sample } & \multicolumn{5}{|c|}{ Out-of-sample } \\
\hline & & RW & Naive & $\mathrm{NN}$ & Asset & $(\%)$ & RW & Naive & $\mathrm{NN}$ & Asset & $(\%)$ \\
\hline & Win. Trade & 0.33 & 0.38 & 0.49 & 0.62 & 0.60 & 0.00 & 0.28 & 0.43 & 0.45 & 0.48 \\
\hline \multirow[t]{6}{*}{ PG } & Annual. Ret & 11.94 & 5.69 & 13.24 & 26.37 & 25.63 & 8.26 & -6.22 & 4.38 & 0.04 & 11.95 \\
\hline & Cum. Ret & 103.17 & 49.15 & 114.35 & 227.78 & 221.39 & 17.84 & -13.43 & 9.46 & 0.08 & 25.80 \\
\hline & Annual. Vol & 33.99 & 17.49 & 41.25 & 25.15 & 23.37 & 0.00 & 9.04 & 10.71 & 2.30 & 9.68 \\
\hline & Sharpe Ratio & 0.35 & 0.33 & 0.32 & 1.05 & 1.10 & Inf & -0.69 & 0.41 & 0.02 & 1.23 \\
\hline & Num. Trade & 6.00 & 547.00 & 109.00 & 310.00 & 327.00 & 1.00 & 144.00 & 108.00 & 23.00 & 38.00 \\
\hline & Win. Trade & 0.17 & 0.36 & 0.56 & 0.66 & 0.59 & 1.00 & 0.31 & 0.54 & 0.43 & 0.63 \\
\hline \multirow[t]{6}{*}{ UTX } & Annual. Ret & 19.07 & 6.85 & 5.57 & 19.66 & 34.75 & 12.21 & 7.11 & -1.56 & 5.87 & 11.46 \\
\hline & Cum. Ret & 164.72 & 59.14 & 48.12 & 169.88 & 300.23 & 26.36 & 15.34 & -3.38 & 12.67 & 24.74 \\
\hline & Annual. Vol & 0.00 & 23.58 & 25.43 & 24.62 & 26.24 & 0.00 & 10.31 & 8.34 & 8.10 & 9.30 \\
\hline & Sharpe Ratio & Inf & 0.29 & 0.22 & 0.80 & 1.32 & Inf & 0.69 & -0.19 & 0.72 & 1.23 \\
\hline & Num. Trade & 1.00 & 558.00 & 414.00 & 356.00 & 280.00 & 1.00 & 141.00 & 106.00 & 70.00 & 29.00 \\
\hline & Win. Trade & 1.00 & 0.38 & 0.53 & 0.65 & 0.65 & 1.00 & 0.34 & 0.53 & 0.50 & 0.62 \\
\hline \multirow[t]{6}{*}{ WMT } & Annual. Ret & 13.91 & 5.07 & 13.03 & 27.85 & 33.34 & -9.83 & 2.72 & -10.08 & -1.14 & 3.53 \\
\hline & Cum. Ret & 120.18 & 43.79 & 112.58 & 240.56 & 288.01 & -21.21 & 5.87 & -21.75 & -2.47 & 7.63 \\
\hline & Annual. Vol & 49.62 & 21.84 & 26.67 & 23.89 & 26.99 & 0.00 & 10.00 & 12.55 & 11.77 & 11.74 \\
\hline & Sharpe Ratio & 0.28 & 0.23 & 0.49 & 1.17 & 1.24 & $-\operatorname{Inf}$ & 0.27 & -0.80 & -0.10 & 0.30 \\
\hline & Num. Trade & 16.00 & 564.00 & 236.00 & 507.00 & 392.00 & 1.00 & 137.00 & 16.00 & 116.00 & 134.00 \\
\hline & Win. Trade & 0.06 & 0.33 & 0.52 & 0.58 & 0.56 & 0.00 & 0.39 & 0.44 & 0.57 & 0.51 \\
\hline
\end{tabular}

Figure 1 shows the average accumulated returns of the 23 assets for the out-of-sample period (544 days), with and without transaction costs. In average, the STARX-Tree model had the better automatic trading results, followed by the random walk model. The difference between the average accumulated returns of STARX-Tree and random walk models is $0.62 \%$, without transaction costs, and $0.48 \%$, with transaction costs.

\section{Leverage}

In order to perform the returns leverage analysis, we use the realized volatility forecast, evaluated using the STARX-Tree methodology, as described in Scharth and Medeiros (2009). We consider an interest rate of 12\% per year.

Figure 2 presents the average accumulated returns of the 23 assets out-of-sample and the average accumulated leveraged returns, considering a maximum financial loss of $10 \%$ with $10 \%$ of probability. In this analysis we do not consider the transaction cost. The model that presents more gain with the leverage possibility is the naive approach. We varied the maximum financial loss in the range of $10 \%-60 \%$, but the STARX-Tree model didn't have good results.

\section{Test of Predictive Accuracy}

In order to measure the statistical significance of the differences between the forecast errors of competing models we employ the modified Diebold-Mariano test, a more robust version of the Diebold and Mariano (1995) approach, proposed by Harvey et al. (1997). Denote $e_{1 t}$ and $e_{2 t}, t=1, \ldots, T$ the 1 -step-ahead forecast error from our STARX-Tree model and the reference model. We tested the three models with the best statistical results as reference, random walk, naive and ARX. 


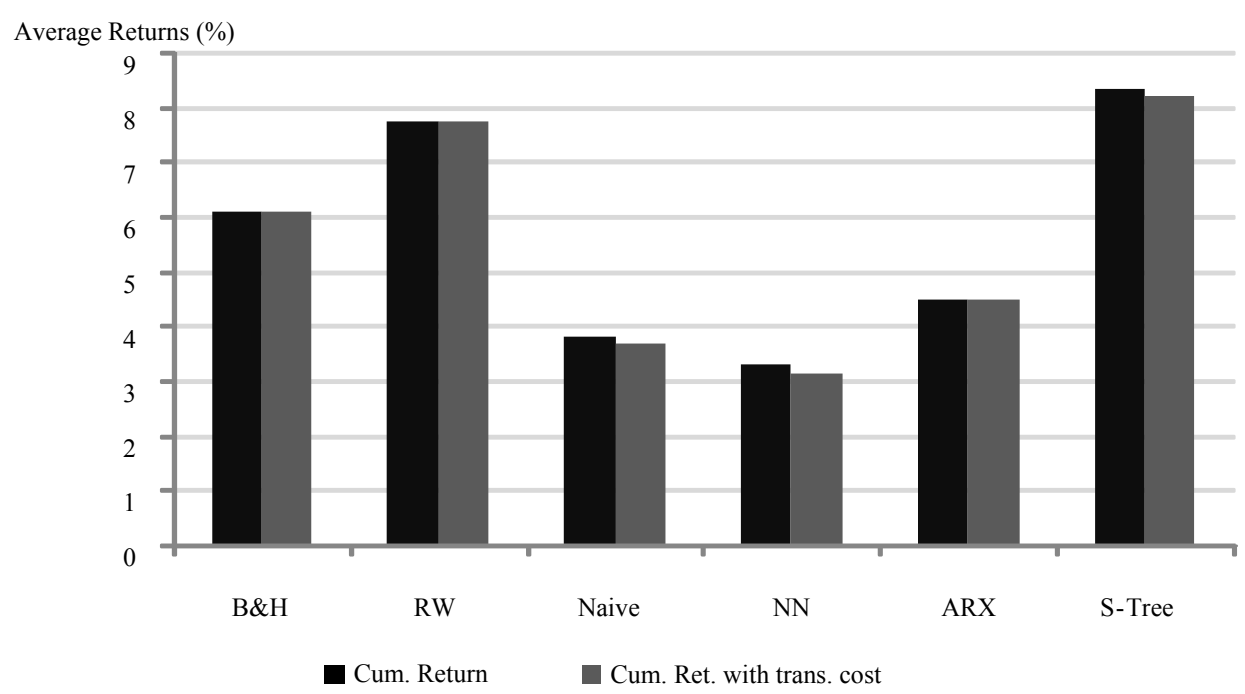

Figure 1. Average accumulated returns of the 23 assets for the out-of-sample period.

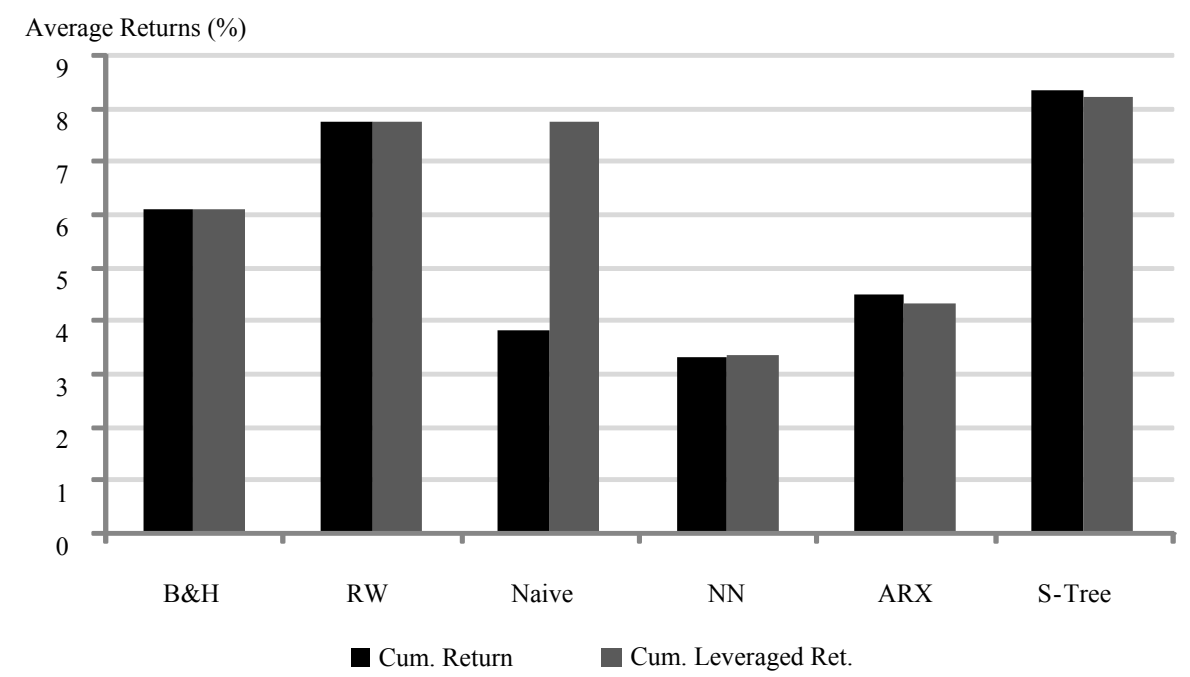

Figure 2. Average accumulated returns of the 23 assets out-of-sample and the average accumulated leveraged returns.

Define the loss function $d_{t}=g\left(e_{1 t}\right)-g\left(e_{2 t}\right)$ and test the null hypothesis $E\left[d_{t}\right]=0$ with the modified Diebold-Mariano statistic. We apply the test with different functions $g(e)$ : absolute error $\left(g\left(e_{i t}\right)=\left|e_{i t}\right|\right)$ and squared error $\left(g\left(e_{i t}\right)=e_{i t}^{2}\right)$. As our main goal is to measure the financial results so we apply the test using a financial loss function. Let $\hat{y}_{i t}$ be the return forecast of model $i$, the function $g$ is defined as:

$$
g\left(\hat{y}_{i t}\right)= \begin{cases}0 ; & \text { if } \hat{y}_{i t} \leq 0 \\ y_{i t} ; & \text { if } \hat{y}_{i t}>0\end{cases}
$$

Therefore, $g\left(\hat{y}_{i t}\right)$ is a vector formed by the returns after the trading, determined by the signal of the forecasts of the returns.

We tested the null hypothesis of "equal accuracy" of the STARX-Tree model in comparison to the random walk model, the naive approach and the ARX model. Table 10 shows the number of series the test rejected the null hypothesis of equality of forecast errors (absolute and squared) and accumulated returns, i.e., series in which the STARX-Tree 1-step-ahead forecast error are lower than the those from the reference model, or the 
STARX-Tree accumulated returns of the out-of-sample period are higher than the those from the reference model. We used a 5\% significance level.

Table 10

No. of Series in Which the STARX-Tree Outperforms the Reference Model

\begin{tabular}{llll}
\hline \multirow{2}{*}{ Reference } & Absoluteerror & Squarederror & Cum. return \\
\cline { 2 - 4 } & S-Tree & S-Tree & S-Tree \\
\hline RW & 0 & 0 & 3 \\
Naive & 22 & 22 & 3 \\
ARX & 7 & 8 & 2 \\
\hline
\end{tabular}

The test did not reject the null hypothesis of equality of forecast errors of the STARX-Tree and random walk models for any assets series. And, with respect to the accumulated returns, the test only rejected the null hypothesis in three series at $5 \%$ significance level. But this does not mean that the forecast errors of the reference model are lower than those from the STARX-Tree model in 23 series, or that the accumulated returns of the reference are higher than those from the STARX-Tree in 20 series. This can be seen by applying the test using the STARX-Tree model as a reference, i.e., we tested if the random walk, naive and ARX forecast errors are lower than the STARX-Tree forecast errors, and if the random walk, naive and ARX accumulated returns are higher than the STARX-Tree accumulated returns.

Table 11 presents the number of series the "reverse" test rejected the null hypothesis of equality of forecast errors (absolute and squared) and accumulated returns at 5\% significance level. Now the reference is the STARX-Tree model.

Table 11

No. of Series in Which Each Model Outperforms the STARX-Tree

\begin{tabular}{|c|c|c|c|c|c|c|c|c|c|}
\hline \multirow{2}{*}{ Reference } & \multicolumn{3}{|c|}{ Absoluteerror } & \multicolumn{3}{|c|}{ Squarederror } & \multicolumn{3}{|c|}{ Cum. return } \\
\hline & $\mathrm{RW}$ & Naive & ARX & RW & Naive & ARX & RW & Naive & $\mathrm{ARX}$ \\
\hline S-Tree & 14 & 0 & 4 & 11 & 0 & 3 & 0 & 0 & 0 \\
\hline
\end{tabular}

Tables 10 and 11 indicate that random walk model outperforms STARX-Tree model with respect to the forecast errors. However, for all series, no model was superior to the STARX-Tree in terms of the financial criterion.

\section{Conclusions}

In this paper we presented the proposal of a model to forecast the returns of the U.S. Stock Market. This model has the advantage of dealing with nonlinear complex structures with the simplicity of a binary decision tree.

Elements of classical statistical inference are used to build the model. The specification is done through a sequence of LM-type tests and the parameters are estimated by Nonlinear Least Squares.

The main goal of this paper was to realize automatic trading using forecasts signal of the returns of 23 assets of the Dow Jones Industrial Average. The random walk model presented better statistical results than the STARX-Tree model. However, in the average, the proposed model outperformed the others in the financial 
criteria, even taking into account the transaction costs. In the test of predictive accuracy, the STARX-Tree model outperformed the others in some series but no model outperformed the STARX-Tree, in terms of the financial criterion. The leverage analysis didn't result in financial gains to the STARX-Tree model.

Another interesting analysis is the study of the selected transition variables. We can see that the volatility changes and the technical analysis indicators have nonlinear effect on return's series.

\section{References}

Andersen, T., Bollerslev, T. F., Diebold, X., \& Labys, P. (2003). Modeling and forecasting realized volatility. Econometrica, 71, $579-625$.

Barndorff-Nielsen, O., \& Shephard, N. (2002). Econometric analysis of realized volatility and its use in estimating stochastic volatility models. Journal of the Royal Statistical Society B, 64, 253-280.

Barndorff-Nielsen, O., Hansen, P., Lunde, A., \& Shephard, N. (2008). Designing realized kernels to measure the ex-post variation of equity prices in the presence of noise. Econometrica, 76, 1481-1536.

Biais, B., Glosten, L., \& Spatt, C. (2005). Market microstructure: A survey of microfoundations, empirical results, and policy implications. Journal of Financial Markets, 8, 217-264.

Breiman, L., Friedman, J., Olshen, R., \& Stone, C. J. (1984). Classification and regression trees. New York: Belmont Wadsworth Int. Group.

Chan, K., \& Tong, H. (1986). On estimating thresholds in autoregressive models. Journal of Time Series Analysis, 7, 179-190.

Da Rosa, J. C., Veiga, A., \& Medeiros, M. (2008). Tree structured smooth transition regression models. Computational Statistics and Data Analysis, 52, 2469-2488.

Diebold, F. X., \& Mariano, R. S. (1995). Comparing predictive accuracy. Journal of Business and Economic Statistics, 13, $253-263$.

Dunis, C., \& Williams, M. (2002). Modelling and trading the EURO/USD exchange rate: Do neural network models perform better? In Derivative Use, Trading \& Regulation, 8/3, 211-239. A fuller version appears as Ch. 1 In C. Dunis, P. Naim, \& L. Laws, (Eds.), Applied quantitative methods for trading and investment. John Wiley \& Sons, Chichester, UK, 2003.

Granger, C., \& Teräsvirta, T. (1993). Modelling nonlinear economic relationships. Oxford: Oxford University Press.

Harvey, D., Leybourne, S., \& Newbold, P. (1997). Testing de equality of prediction mean squared errors. International Journal of Forcasting, 13, 281-291.

Hillebrand, E., \& Medeiros, M. (2010). The benefits of bagging for forecast models of realized volatility. Econometric Reviews, 29, 571-593.

LeBaron, B. (1999). Technical trading rule profitability and foreign exchange intervention. Journal of International Economics, 49 , 125-143.

Luukkonen, R., Saikkonen, R., \& Teräsvirta, T. (1988). Testing linearity against smooth transition autoregressive models. Biometrika, 75, 491-499.

Lyons, R. K. (2001). The microstructure approach to exchange rates. Massachuttes: The MIT Press.

Mcaleer, M., \& Medeiros, M. (2008). Realized volatility: A review. Econometric Reviews, 27, 10-45.

Neely, C. J., \& Weller, P. A. (2003). Intraday technical trading in the foreign exchange market. Journal of International Money and Finance, 22, 223-237.

Neely, C. J., Weller, P. A., \& Dittmar, R. (1997). Is technical analysis in the foreign exchange market profitable? A genetic programming approach. Journal of Financial and Quantitative Analysis, 32, 405-426.

Qi, M., \& Wu, Y. (2002). Technical trading-rule profitability, data snooping, and reality check: Evidence from the foreign exchange market. Mimeo, Rutgers Business School.

Scharth, M., \& Marcelo, C. M. (2009). Asymmetric effects and long memory in the volatility of DJIA stocks. International Journal of Forecasting, 25, 304-327.

Teräsvirta, T. (1994). Specification, estimation, and evaluation of smooth transition autoregressive models. Journal of the American Statistical Association, 89, 208-218. 